Portland State University

PDXScholar

Spring 6-10-2019

\title{
Infinite Photovoltaic Solar Arrays: Considering Flux of Momentum and Heat Transfer
}

Andrew D. S. Glick

Portland State University

Follow this and additional works at: https://pdxscholar.library.pdx.edu/open_access_etds

Part of the Mechanical Engineering Commons

Let us know how access to this document benefits you.

\section{Recommended Citation}

Glick, Andrew D. S., "Infinite Photovoltaic Solar Arrays: Considering Flux of Momentum and Heat Transfer" (2019). Dissertations and Theses. Paper 5113.

https://doi.org/10.15760/etd.6992

This Thesis is brought to you for free and open access. It has been accepted for inclusion in Dissertations and Theses by an authorized administrator of PDXScholar. Please contact us if we can make this document more accessible: pdxscholar@pdx.edu. 
Infinite Photovoltaic Solar Arrays:

Considering Flux of Momentum and Heat Transfer

\title{
by
}

\author{
Andrew D. S. Glick
}

A thesis submitted in partial fulfillment of the requirements for the degree of

\author{
Master of Science \\ in \\ Mechanical Engineering
}

Thesis Committee:

Raúl Bayoán Cal, Chair

Gerald Recktenwald

Derek Tretheway

Portland State University

2019 


\begin{abstract}
Large scale solar farms supply an increasing amount of the worlds electricity supply. However, in order to reach cost parity with fossil fuels, further reductions are necessary. Towards this end, photovoltaic (PV) panel cooling becomes increasingly important; high temperatures both decrease efficiency and panel lifetime. To better understand, characterize, and exploit the natural convective cooling of utility scale solar farms, a model solar farm was created. Using both thermal measurements and particle image velocimetry to characterize heat transfer and velocity fields, wind tunnel experiments were conducted using the model solar farm. Three parameters were examined for their effect on heat transfer and the flow field: Reynolds number, inflow turbulence intensity (TI), and PV inclination angles. Results show that increasing inflow turbulence improved both upper and lower surface heat transfer by $7 \%$, and lower surface increases on order of $100 \%$ were demonstrated in both the flow field and heat transfer with changes in angle inclination. Results suggest that significant farm level temperature reductions are possible.
\end{abstract}




\section{Acknowledgements}

The author would like to thank Raúl Bayoán Cal for guidance through the Masters program, Portland State University's Department of Mechanical and Materials Engineering for funding and support, The United States Department of Energy Sunshot Initiative for funding and support, the members of the Wind Energy and Turbulence Lab including Dr. Naseem Ali, Dr. Juliaan Bossuyt, and Sarah Smith for many hours of experimental work and discussion of results, Dr. Gerald Recktenwald of PSU for guidance and much shared expertise on experimental design and heat transfer, Ryan Scott for help with experimental fabrication, Bianca Viggiano for lots of help early on with experimental fabrication and PIV, Dr. Derek Tretheway for reviewing and providing feedback on my thesis, Dr. Marc Calaf and the team at University of Utah for many conversations regarding the experimental design and analysis, as well as Dr. Scott Schreck for guidance and research training during my time as an undergraduate researcher at National Renewable Energy Laboratory. Lastly but not least-ly, I would like to thank Karina Kreider, for never-ending support as I worked towards this degree. 


\section{Contents}

Abstract

Acknowledgements

List of Tables $\quad$ v

List of Figures vi vi vis

Nomenclature viii

1 Introduction 1

1.1 Background ............................ 1

1.2 Importance of wind speed on PV farm and cell temperatures . . . . . 4

1.3 Related applications using arrays of heated elements . . . . . . . . . . 6

2 Theory and Data Reduction 10

2.1 Heat Transfer Theory and Data Reduction . . . . . . . . . . . . . . . 10

2.2 Momentum Theory . . . . . . . . . . . . . . . . . 15

3 Experimental Methods $\quad 17$

3.1 Experimental Facility . . . . . . . . . . . . . . . 17 
3.2 Solar Farm Fabrication and Heating . . . . . . . . . . . . . . . 20

3.3 Uncertainty Analysis . . . . . . . . . . . . . . . 27

3.4 Data Measurement and Acquisition . . . . . . . . . . . . . . 27

3.4.1 Temperature Measurement and Acquisition . . . . . . . . . . . 27

3.4 .2 Particle Image Velocimetry . . . . . . . . . . . . . . . . . . . . 28

3.5 Test Matrix ... . . . . . . . . . . . . . 33

4 Results $\quad 36$

4.1 Reynolds Number and Power Density Effects . . . . . . . . . . . . 37

4.1.1 Flow field statistics . . . . . . . . . . . . . . . . 41

4.2 Turbulence Intensity Effects . . . . . . . . . . . . . . . . . . 48

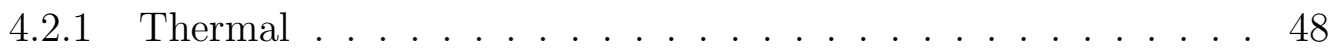

4.2.2 Flow field statistics . . . . . . . . . . . . . . 50

4.3 Panel Inclination Angle Effects . . . . . . . . . . . . . . 53

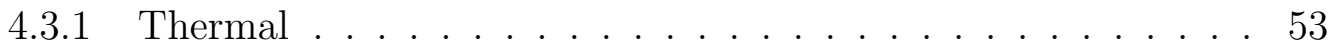

4.3.2 Flow field statistics . . . . . . . . . . . . . 57

5 Conclusions $\quad 62$

$\begin{array}{lll}6 & \text { Future Work } & 64\end{array}$

$\begin{array}{ll}\text { Bibliography } & 65\end{array}$

$\begin{array}{ll}\text { Appendices } & 70\end{array}$

A Data Reduction Equations ................. 71

B Uncertainty Analysis .................. 71 


\section{List of Tables}

3.1 Test matrix: parameter values used in experiments . . . . . . . . 34

4.1 Nusselt Number correlation equations . . . . . . . . . . . . . . . . . . 40 


\section{List of Figures}

2.1 Energy Balance Heat Transfer Model, Panel Section View . . . . . . . 11

2.2 Heat Resistance Circuit . . . . . . . . . . . . . . . . . . . . 14

3.1 Schematic of wind tunnel experimental setup . . . . . . . . . 18

3.2 Inflow statistics . . . . . . . . . . . . . . . . . . 21

3.3 Schematic and photographic side views of model solar panels . . . . . 23

3.4 Individual Panel . . . . . . . . . . . . . . . . . . . 24

3.5 Model solar panel planform views . . . . . . . . . . . . . 25

3.6 Model solar farm in experimental wind tunnel . . . . . . . . . 26

3.7 Data Acquisition Setup . . . . . . . . . . . . . . . 28

3.8 Solar Farm shown from PIV camera, perspective view. . . . . . . . . 29

3.9 Velocity spatial contour plot . . . . . . . . . . . . 30

3.10 Streamwise-averaged profiles of normalized streamwise velocity for three different spatial averaging windows. . . . . . . . . . . . . 31

3.11 Streamwise-averaged profiles of normalized vertical velocity profiles calculated with different averaging windows . . . . . . . . . 32

3.12 Streamwise-averaged profiles of normalized Reynolds shear stress profiles calculated with different averaging windows . . . . . . . . . . 33

4.1 Adiabatic heat transfer for upper and lower surfaces of Row 8 . . . 37 
4.2 Comparison of Nusselt number to literature . . . . . . . . . . . . 40

4.3 Reynolds number effect on normalized mean velocity profiles . . . . . 42

4.4 Reynolds number effect on normalized Reynolds stresses . . . . . . . 45

4.5 Input power level effect on normalized mean velocity profiles . . . . . 46

4.6 Input power level effect on normalized Reynolds stresses . . . . . . . 47

4.7 Turbulence effects on $N u_{a d, u p}$ and $N u_{a d, l o} \ldots \ldots \ldots \ldots$

4.8 Inflow turbulence effects on normalized mean velocity profiles . . . . 50

4.9 Inflow turbulence effects on normalized Reynolds stresses . . . . . . . 51

4.10 Panel inclination angle effect on $N u_{a d, u p}$ and $N u_{a d, l o} \ldots \ldots$. . . . . 53

4.11 Surface Temperature Variations between $+30^{\circ}$ and $-30^{\circ}$ at $q_{f}^{\prime \prime}=0.42 \quad$. 56

4.12 Panel inclination angle effect on normalized mean velocity profiles . . 58

4.13 Panel inclination angle effect on normalized Reynolds stresses . . . . 61 


\section{Nomenclature}

$\sigma \quad$ Stephan Boltzmann Constant

$\theta \quad$ Temperature of the fluid at a point in the $x-y$ plane

A Area of panel perpendicular to the direction of heat flux

$i \quad$ Current through resistive heaters

$V \quad$ Voltage across an individual panel

$A G_{H i}$ Active grid upper setting

$A G_{L o}$ Active grid lower setting

$\epsilon_{l o} \quad$ Module lower surface emissivity

$\epsilon_{u p} \quad$ Module upper surface emissivity

$F_{\text {lo_ceil }}$ Radiation view factor from panel lower surface to tunnel ceiling

$F_{l o_{-} f l r}$ Radiation view factor from panel lower surface to tunnel floor

$F_{\text {lo_pan }}$ Radiation view factor from panel lower surface to rear nearest panel

$F_{\text {up_ceil }}$ Radiation view factor from panel upper surface to tunnel ceiling 
$F_{u p_{-} f l r}$ Radiation view factor from panel upper surface to tunnel floor

$F_{\text {up_pan }}$ Radiation view factor from panel upper surface to rear nearest panel

$h_{a d, l o}$ Module lower surface average adiabatic convective heat transfer coefficient

$h_{a d, u p}$ Module upper surface average adiabatic convective heat transfer coefficient

$h_{a d} \quad$ Adiabatic convective heat transfer coefficient

$h_{c} \quad$ Convective heat transfer coefficient

$\alpha \quad$ Inclination angle between panel and the horizontal aligned with the direction of flow

$k_{\text {air }} \quad$ Thermal conductivity of Air

$k_{a l} \quad$ Thermal conductivity of 6061 aluminum

$k_{\text {ins }} \quad$ Thermal conductivity of Aerogel Insulation

$L_{a l} \quad$ Thickness of aluminum panel

$L_{\text {ins }} \quad$ Thickness of Aerogel Insulation

$N u_{a d, l o}$ Adiabatic lower surface Nusselt number

$N G \quad$ Inflow setting using no grid

$N u_{a d, u p}$ Adiabatic upper surface Nusselt number

$P G \quad$ Inflow setting using grid installed but aligned with wind and motionless

$q_{c o n v, l o}^{\prime \prime}$ Convective heat flux from the lower module surface 
$q_{c o n v, l o}$ Convective heat from the lower module surface

$q_{\text {conv,up }}^{\prime \prime}$ Convective heat flux from the upper module surface

$q_{c o n v, u p}$ Convective heat from the upper module surface

$q_{e}^{\prime \prime} \quad$ Electric heat flux supplied to the kapton heater

$q_{e} \quad$ Electric heat supplied to the kapton heater

$q_{l o}^{\prime \prime} \quad$ Heat flux passing from the heater through the lower module surface

$q_{l o} \quad$ Heat passing from the heater through the lower module surface

$q_{f}^{\prime \prime} \quad$ Nondimensionalized power input $q_{e}^{\prime \prime} / q_{0}^{\prime \prime}$

$q_{0}^{\prime \prime} \quad$ Direct Solar Radiation at earths surface

$q_{\text {rad,lo,ceil }}^{\prime \prime}$ Radiative heat flux losses from the lower module surface to the tunnel ceiling

$q_{r a d, l o, f l r}^{\prime \prime}$ Radiative heat flux losses from the lower module surface to the tunnel floor

$q_{\text {rad,lo,pan }}^{\prime \prime}$ Radiative heat flux losses from the lower module surface to the rear side of the adjacent panels

$q_{\text {rad,lo }}^{\prime \prime}$ Total Radiative heat flux losses from the lower module surface

$q_{\text {rad,up,ceil }}^{\prime \prime}$ Radiative heat flux losses from the upper module surface to the tunnel ceiling

$q_{\text {rad,up,flr }}^{\prime \prime}$ Radiative heat flux losses from the upper module surface to the tunnel floor 
$q_{\text {rad,up,pan }}^{\prime \prime}$ Radiative heat flux losses from the upper module surface to the rear side of the adjacent panels

$q_{\text {rad,up }}^{\prime \prime}$ Total Radiative heat flux losses from the upper module surface

$q_{u p} \quad$ Heat passing from the heater through the upper module surface

$q_{u p}^{\prime \prime} \quad$ Heat flux passing from the heater through the upper module surface

$R_{a l} \quad$ Thermal resistance of Aluminum layer of module

$R_{b c k} \quad$ Thermal resistance from kapton heater to lower module surface

$R_{f n t}$ Thermal resistance from kapton heater to upper module surface

$R_{\text {insl }}$ Thermal resistance of Aerogel Insulation layer of module

$T_{a d, l o}$ Module lower surface average adiabatic temperature

$T_{a d, u p}$ Module upper surface average adiabatic temperature

$T_{a m b}$ Wind tunnel ambient temperature

$T_{\text {bulk }}$ Wind tunnel bulk mean temperature

$T_{\text {ceil }}$ Wind tunnel ceiling temperature

$T_{\text {floor }}$ Wind tunnel floor temperature

$T_{H t} \quad$ Kapton Heater temperature

$T_{l o} \quad$ Module lower surface average adiabatic temperature

TI Incoming freestream turbulence intensity 
$T_{u p} \quad$ Module upper surface average adiabatic temperature

$U_{\infty} \quad$ Test section freestream velocity

$\Phi \quad$ Angle between incoming wind and panel row

B Element Height (tunnel floor to lowest edge of panel)

$\mathrm{H} \quad$ Height of top of panel from tunnel floor (30 deg case)

L Length of panel, along incline

LCOE Levelized Cost of Energy

LS Streamwise Spacing between elements, or longitudinal, (front to front distance)

Module Model solar panel structure, consisting of heater, insulation and aluminum surfaces 


\section{Chapter 1}

\section{Introduction}

\section{$1.1 \quad$ Background}

It is well documented that the operating temperature is a crucial operating parameter of photovoltaic (PV) solar cells, having a significant effect on both panel efficiency and panel lifetime. In a 2009 review, Skoplaki et al. presented efficiency correlations demonstrating that module efficiency decreased by $0.2-0.5 \%$ per degree Celsius [1] [2]. PV modules are tested at $25^{\circ} \mathrm{C}$, but operate closer to $44^{\circ} \mathrm{C}$ and upwards to $65^{\circ} \mathrm{C}$, which decreases the efficiency $5-12 \%$ compared to module tested value [3]. Further, increased cell temperature is also responsible for accelerated aging of PV modules, due to common thermally activated degradation mechanisms [4]. For crystalline silicone PV cells, the aging rate doubles for every $10^{\circ} \mathrm{C}$ increase in operating temperature [5].

These temperature efficiency relationships imply that the levelized cost of energy (LCOE) for solar PV is directly related to module efficiency, where a $5 \%$ efficiency gain means 5-10\% reduction in LCOE [6]. Presently, efforts to reduce LCOE through efficiency improvements have centered around improving individual cell efficiencies at $25^{\circ} \mathrm{C}$. However, further reducing the optical and electrical losses of individual 
cells is becoming increasingly costly [7]. This work approaches the same goal through an underexplored avenue; passively improving waste heat rejection at the solar farm level. By examining and modifying the fluid flow through the farm in order to increase thermal mixing and array thermal heterogeneity, convective heating can be improved and PV module efficiency substantively increased.

A wide variety of approaches have been used to reduce PV module cell temperature. Two general strategies exist towards this end: 1) Decreasing waste heat generation and 2) Increasing waste heat rejection [8]. Within those categories, both passive and active techniques are employed.

Decreasing waste heat generation has long been a focus of solar research and engineering. Efforts have been largely focused on improving electrical conversion efficiency, reflecting unusable photons, and altering surface emissivity. The National Renewable Energy Laboratory chart of the best research cell electrical conversion efficiencies chronicles the rise in efficiencies of a variety of current and emerging technologies. Increasing cell efficiency directly decreases waste heat generation by capturing as electricity what would have been converted to heat.

Further methods of decreasing waste heat generation include advanced reflectance and radiation altering techniques. Optical modifications have the potential to decrease the panel temperature as much as $4^{\circ} \mathrm{C}$ using ideal sub-bandgap reflectors or infrared reflector films [8]. Increasing surface emissivity off the front and rear surfaces to increase the radiative heat transfer from the panels has also been explored, though this has shown only minimal temperature reduction potential [8]. Radiative cooling also shows promise, wherein a layer is placed on the PV surface which is transparent over solar wavelengths but strongly emissive over thermal wavelengths, 
thus rejecting waste heat to outer space [9].

Increasing waste heat rejection has invited a plethora of creative techniques of varying degrees of economic and practical use. Forced air cooling has been used in photovoltaic/thermal (PV/T) systems, which duct the PV thermal losses for use in nearby buildings [10]. Teo et al. showed that the efficiency of the PV panel increased $4 \%$ with the use of forced air cooling, reaching a total combined efficiency of $50 \%$, including the direct thermal gains [11]. Yang showed that the use of forced liquid cooling increased the overall efficiency to as high as $70 \%$ [12]. Despite these gains, $\mathrm{PV} / \mathrm{T}$ systems have not found traction in large arrays due to high cost and the lack of nearby buildings and uses for thermal energy at the rural locations of most large solar arrays. Further barriers include the negative effect to LCOE that occurs using active techniques (e.g. fan to push air or pump to move water).

Further explorations into increasing waste heat rejection include modification of module thermal conductivity and phase change materials, and a summary of both active and passive efforts to enhance waste heat rejection was compiled by Hasanuzzaman et al. [2][8]. A review of the techniques to reduce operating temperature showed many active techniques, however these approaches must always be weighed against additional operations and maintenance costs. The proposed technique here examines a passive technique that should not degrade with time or require extra maintenance.

Within the waste heat rejection strategy, farm level convection enhancements remain unexplored. Two large bodies of study suggest opportunities in this approach. Firstly, much work has demonstrated the importance of wind speed and other environmental conditions to both farm efficiency and individual panels. Secondly, 
other applications using arrays of heated elements have shown significant cooling improvements through optimization of array layout [13].

\subsection{Importance of wind speed on PV farm and cell temperatures}

Energy production models for PV system all include cell temperature as a critical parameter. De Soto et al. presented a five parameter power prediction model using solar irradiation and cell temperature as the critical input parameters [14]. Module cell temperature is itself a function of a wide range of operating parameters. Earlier solar farm power production models developed by King et al. have taken overhead wind speed at $10 \mathrm{~m}$, wind direction, ambient temperature and thermal response time into account. King et al. showed a cell temperature dependence of $1.33^{\circ} \mathrm{C}$ per $1 \mathrm{~m} / \mathrm{s}$ increase in overhead wind speed, and noted that using temperature to determine module output, though important, is not standardized [15].

Further studies have demonstrated that the cell temperature is dependent on local wind speed, solar radiation flux, dust, and humidity as well as system-dependent parameters such as glazing and absorptance [16][17]. Standing out among these variables is the overhead wind speed, which has a sizeable impact on convective cooling rates over each individual panel as well as the overall farm [18]. In a review of the pertinent correlations, Skoplaki et al. noted that cell temperature is extremely sensitive to wind speed. However, to date most of the correlations presenting cell temperature as an explicit function of wind speed are site specific [19]. While the importance of wind speed in solar farm efficiency has been established, no thermal optimization of solar arrays through layout design has been pursued.

The individual module temperature has been examined further in the case of flat 
plate solar collectors, which in contrast to PV are used not for electricity generation but to directly heat water. The relationship between convective heat transfer and overhead wind speed has been explored in order to properly characterize the heat losses to natural convective cooling. Linear relationships have been found between the top loss heat transfer coefficient and the overhead wind speed, beginning with McAdams et al. and corroborated by Sparrow et al. [20][21][22]. Much of this work was done in controlled, indoor environments. However, outdoor experiments have shown higher convection due to incoming turbulence and aerodynamic shedding off nearby features [23][24]. Silverman et al. noted the lack of appropriate functional relationships for solar farm temperature modeling and analysis [8].

Various parameters have been examined, including wind direction and inclination angle, where differing results on the effect of inclination angle have been found [25][26]. Hobbi et al. showed increases in convective heat transfer from the panel to the heated liquid through the use of heat enhancement devices in the liquid channel [27]. Variations in convective heat transfer coefficient due to angle of attack, indicative of end effects, have been shown to be negligible for panels larger than an aspect ratio of $2.5[20]$.

The studies examining cooling of individual solar panels mentioned thus far have mostly been concerned with forced convection, however a wide body of work exists examining the natural convection from vertical, horizontal, and inclined heated plates. With specific applications to solar power, the effect of natural convection on two parallel plates inclined between $0^{\circ}$ and $75^{\circ}$ from the horizontal has been recorded [28]. Much of the early work on understanding convective heat transfer in solar modules was aimed at solar flat plate collectors and thus attempting to de- 
crease the free convection heat transfer. Further, only two panels were considered, and no combination of free and forced convection.

\subsection{Related applications using arrays of heated elements}

Although not exclusively focused on solar array heating, considerable applicable work in single phase convection has been performed to understand the cooling of arrays of electronic components on printed circuit board assemblies, and arrays of pin fin heat sinks for turbines [29].

Sparrow and Niethammer performed work to demonstrate heat transfer enhancements with forced air convection over heated rectangular bodies in a duct [13]. Using napthalene sublimation, element removal increased the heat transfer coefficient in all surrounding bodies, reaching as high as a $40 \%$ increase for the element directly upstream. Further research demonstrated increases ranging from 7-40\% due to increased streamwise spacing, staggered rows, and barriers. Implanted barriers showed the greatest increase to convective heat transfer, ranging from 39\%-67\% from channel Reynolds numbers 2000-7000. Experiments used an element thickness to channel height ratio of $3 / 8$, a much higher value than would be used to mimic solar arrays in the atmospheric boundary layer (ABL) [13].

Numerical work further explored the potential of using barriers to increase convective heat transfer. Wu et al. used an angled oblique plate above the height of the elements to determine the array flow characteristics and the resulting change in Nusselt number. Mixed convection was modeled, in contrast to many earlier numerical studies looking at purely forced convection. Maximum Nusselt number increases of $39 \%$ were recorded at an oblique plate angle of $60 \%$ [30]. This idea was 
further developed by Yang with the concept of an oscillating plate to mix the flow, finding enhancements at particular Reynolds numbers and oscillation amplitudes to vary from $37-74 \%$ [31].

Forced liquid cooling showed similar results, demonstrating an increasing heat transfer coefficient as streamwise spacing increased, as layouts were staggered, and as duct height decreased. Garimella and Eibeck measured the heat transfer characteristics over an array of protruding elements mounted in a channel. Governing nondimensional parameters used were streamwise-spacing-to-element-height ratio and the channel-to-element-height ratio. The Nusselt number variation was correlated to the array Reynolds number and the non dimensional streamwise spacing [29]. Huang et al. showed 8-13\% increases in convective heat transfer over arrays in finned tube heat exchangers using a staggered configuration compared to an inline configuration [32]. Test et al. showed that higher incoming turbulence intensity increased the convection heat transfer coefficient [33].

Further heat transfer work using arrays of cubic and diamond fins from a plane wall correlated the row averaged convective heat transfer coefficients in staggered and in-line arrays and found increases ranging from 20-80\% [34]. Heat transfer over the arrays depended on two competing factors: the increase in heat transfer due to direct wake shedding, and the decrease due to elevated bulk mean temperature in the flow due to heat removed from upstream elements.

The above work focused on understanding and augmenting the heat transfer coefficient. Anderson and Moffat challenged the assumption that increased heat transfer coefficient necessarily achieves the underlying goal of element temperature reduction by noting that an increased heat transfer coefficient does not influence 
if it is accompanied by a decrease in temperature gradient [35]. They designed experiments with scoops in between rows of elements to increase the thermal mixing between the bypass flow and the array flow and increase the temperature gradient without necessarily inducing turbulence. Superposition was used to calculate the adiabatic heat transfer coefficient for an array of protruding elements, demonstrating a $50 \%$ reduction in the adiabatic temperature rise and a $19 \%$ reduction in the overall temperature rise [35].

In addition to their heat transfer characteristics, arrays have been characterized for their flow separation and reattachment properties. In large arrays of elements, the flow is divided into array flow and bypass flow, the latter of which increases in thickness over the first 3-4 rows before becoming constant. Greater turbulence intensity was observed in the cavity flow for larger streamwise spacing, and reattachment lengths in the cavity were calculated [36]. When using vortex generators for heated elements in a channel, Wu et al. observed strong coupling between the vortex shedding and buoyancy effects [30]. Young et al. continued this numerical work simulating heated obstacles in a channel with respect to obstacle width, height, and spacing [37]. Decreasing size and increasing relative spacing were found to better transfer heat into the surrounding fluid.

This work was performed with two aims: 1) to determine accurate and generalizable values for convective heat transfer coefficient $\left(h_{c}\right)$ to be used in PV farm and module modeling efforts and 2) to do a comparative analysis considering the effect of Reynolds number, turbulence intensity, and panel inclination angle on the convective cooling of large ground-mounted solar arrays. Improved physics based models will help researchers better understand where they should direct their improvement 
efforts, as well as offer insights into how farm layouts might lead to better efficiencies. Ultimately, these improved efficiencies would reduce cost, leading to greater adoption of solar technologies. This document outlines the theory, experiment, results, and conclusions of this effort. 


\section{Chapter 2}

\section{Theory and Data Reduction}

\subsection{Heat Transfer Theory and Data Reduction}

The convective heat transfer coefficient $h_{c}$ is defined as the heat flux $q_{e}^{\prime \prime}$ across a surface, divided by the thermal potential across that surface $T_{\text {sur }}-T_{\text {ref }}$, where $T_{\text {sur }}$ is the surface temperature and $T_{r e f}$ is a reference temperature,

$$
h_{c}=\frac{q^{\prime \prime}}{T_{r e f}-T_{s u r}} .
$$

Several choices exist for a choice of the reference temperature $T_{\text {ref }}$, namely the bulk mean temperature of the fluid, the temperature upstream of the array, and the adiabatic surface temperature. An extensive discussion by Moffat and Ortega explores the various methods of choosing the reference temperature [38]. The adiabatic heat transfer coefficient $h_{a d}$ is defined using the adiabatic temperature of the panel as the reference temperature. Adiabatic surface temperature is the surface temperature of an unheated panel when all other upstream panels are heated, and represents the temperature of the thermal wakes produced by upstream elements. This definition allows for better comparison with existing literature and ensures that $h_{c}$ is a function only of geometry and flow, and does not depend on an upstream 
heating pattern [38]. This independence of $h_{c}$ on upstream heating is important in part due to differences between the present study experimental setup (where the lower module surface convects less heat due to insulation) and real solar panels (where the lower surfaces is not insulated).

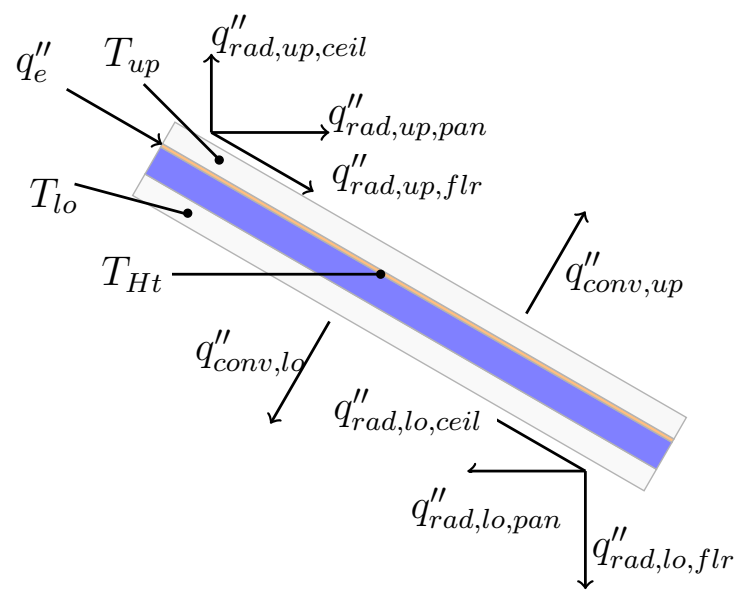

Figure 2.1: Energy Balance Heat Transfer Model, Panel Section View

A steady state model of the panels was developed as shown in Fig. 2.1 and used to calculate the average adiabatic convective heat transfer coefficient on the upper module surface $h_{a d, u p}$ and the lower module surface $h_{a d, l o}$. In this model, $q_{e}^{\prime \prime}$ is the electric heat flux input to the Kapton heater, $q_{\text {conv,up }}^{\prime \prime}$ the convective heat flux loss from the upper module surface, and $q_{c o n v, l o}^{\prime \prime}$ is the convective heat flux loss from the lower module surface facing the floor of the wind tunnel.

The radiation terms $q_{\text {rad,up }}^{\prime \prime}$ and $q_{\text {rad,lo }}^{\prime \prime}$ are the total radiative heat flux losses from the upper and lower module surfaces respectively. These general upper and lower radiative terms are further broken down according to their heat flux to the ceil$\operatorname{ing}\left(q_{\text {rad,up,ceil }}^{\prime \prime}, q_{\text {rad,lo,ceil }}^{\prime \prime}\right)$, adjacent panel $\left(q_{\text {rad,up,pan }}^{\prime \prime}, q_{\text {rad,lo,pan }}^{\prime \prime}\right)$, and floor $\left(q_{\text {rad,up,flr }}^{\prime \prime}\right.$, $\left.q_{r a d, l o, f l r}^{\prime \prime}\right)$ respectively. Upper surface view factors $F_{u p_{-} f l r}, F_{u p_{-} p a n}$, and $F_{\text {up_ceil }}$ were 
approximated as $0.4,0.4$, and 0.2 respectively, while $F_{l o_{-} f l r}, F_{l o \_p a n}$, and $F_{l o_{-} c e i l}$ were estimated as $0.2,0.4$, and 0.4 from visual inspection. Uncertainty in $h_{a d, u p}$ due to view factors was less than 1\%. Full equations are shown in Appendix A.

Heat losses from the sides were estimated using their relative areas to be a maximum of $3.8 \%$, and likely considerably less due to a less exposure to incoming fluid. The basic energy balance equation is then,

$$
q_{e}^{\prime \prime}=q_{u p}^{\prime \prime}+q_{l o}^{\prime \prime}
$$

where the heat loss out the top and bottom surfaces are then defined,

$$
\begin{gathered}
q_{u p}^{\prime \prime}=q_{c o n v, u p}^{\prime \prime}+q_{r a d, u p}^{\prime \prime}, \\
q_{l o}^{\prime \prime}=q_{c o n v, l o}^{\prime \prime}+q_{r a d, l o}^{\prime \prime} .
\end{gathered}
$$

Temperatures shown in the model above include $T_{\text {ceil }}$, the temperature of the upper surface of the wind tunnel, $T_{\text {floor }}$ the temperature of the floor of the wind tunnel, $T_{u p}$ the temperature on the module upper surface, and $T_{l o}$ the temperature on the module lower surface. The temperature of the heater itself, $T_{H t}$ was not measured, but is calculated in the thermal resistance model.

Radiative losses to the ceiling and the floor of the wind tunnel were estimated using upper and lower surface emissivities, $\epsilon_{u p}$ and $\epsilon_{l o}$ of $0.90 \pm 0.04$. For radiation calculations, floor, ceiling, and panels were assumed to be gray bodies with constant ensemble and spatial emissivity values. 
A thermal resistance model of the heat transfer is then used to determine the convection heat flux off of the upper and lower surfaces: $q_{c o n v, u p}^{\prime \prime}$ and $q_{c o n v, l o}^{\prime \prime}$ respectively. Neglecting end losses and assuming spatially uniform thermal conductivities, this heat flux can be modeled as shown in Fig. 2.2, and the heat fluxes off the upper and lower surfaces written as in Equations 2.5 and 2.6,

$$
\begin{gathered}
q_{l o}^{\prime \prime}=\frac{T_{H t}-T_{l o}}{R_{b c k}}, \\
q_{u p}^{\prime \prime}=\frac{T_{H t}-T_{u p}}{R_{f n t}} .
\end{gathered}
$$

In Equations 2.5 and 2.6, $R_{f n t}$ is the thermal resistance from $T_{H t}$ to $T_{u p}$ and $R_{b c k}$ is the thermal resistance from $T_{H t}$ to $T_{l o}$ calculated. Full definitions of each resistance term are included in the appendix. Contact resistances between each layer (aluminum, heater, tape, aerogel, tape, aluminum) were neglected due to the scale of the thermal resistance of the aerogel insulation (near $10 \mathrm{~m}^{2} \mathrm{KW}^{-1}$ ) compared to contact resistances (near $3 \times 10^{-5} \mathrm{~m}^{2} K W^{-1}$ ). Equations 2.2, 2.5, and 2.6 were solved for the upper and lower convective heat flux terms, $q_{u p}$ and $q_{l o}$ respectively.

When steady state conditions were achieved, $h_{a d, u p}$ and $h_{a d, l o}$ were calculated using the simplified model equations above and $q_{c o n v, u p}^{\prime \prime}$ and $q_{c o n v, l o}^{\prime \prime}$ values calculated from the resistance model, and averaged over a $5 \mathrm{~min}$ period. Using $h_{a d, u p}$ and $h_{a d, l o}$, the average Nusselt number of the upper surface of the panel was calculated using the height of the lower edge of the panel from the ground (B), and the thermal conductivity of air at the film temperature $k_{f}$ in the relation 


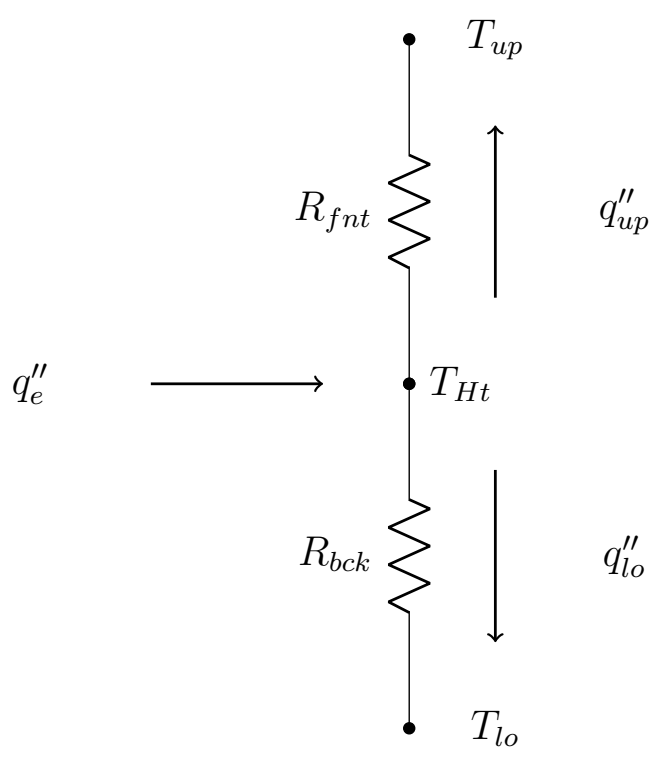

Figure 2.2: Heat Resistance Circuit

$$
N u_{a d, u p}=\frac{h_{a d, u p} B}{k_{f}}
$$

The lower surface $N u_{a d, l o}$ was calculated with the same method using $h_{a d, l o}$. Using the ensemble averaged freestream velocity $U_{\infty}$ and the kinematic viscosity of air $\nu$ the ensemble averaged Reynolds number is defined as:

$$
R_{e}=\frac{U_{\infty} B}{\nu}
$$

All fluid properties were calculated at the film temperature, defined as the average of the adiabatic temperature and the heated surface temperature. 


\subsection{Momentum Theory}

To characterize the turbulent flow, the Reynolds-Averaged Navier-Stokes (RANS) equation was used,

$$
\frac{\partial \overline{u_{i}}}{\partial t}+\overline{u_{j}} \frac{\partial \overline{u_{i}}}{\partial x_{j}}=-\frac{1}{\rho} \frac{\partial \bar{p}}{\partial x_{i}}+\nu \frac{\partial^{2} \overline{u_{i}}}{\partial x_{j}^{2}}-\frac{\partial}{\partial x_{j}}\left[\overline{u_{i}^{\prime} u_{j}^{\prime}}\right]-F_{i}
$$

where $t$ is time, $\rho$ is the density, $p$ is the pressure, $\nu$ is the kinematic viscosity, and $F_{i}$ is the force on the solar panels. The velocity component convention is such that $u, v$, and $w$ correspond to the velocities in the streamwise $(x)$, wall-normal $(y)$, and spanwise $(z)$ directions. The overbar indicates ensemble averaging, and prime indicates fluctuations. For incompressible steady flows sufficiently far from any solid boundaries such that viscous contributions can be neglected, the equations become,

$$
\overline{u_{j}} \frac{\partial \overline{u_{i}}}{\partial x_{j}}=-\frac{1}{\rho} \frac{\partial \bar{p}}{\partial x_{i}}-\frac{\partial}{\partial x_{j}}\left[\overline{u_{i}^{\prime} u_{j}^{\prime}}\right]-F_{i} .
$$

The left side of Eq. 2.11 describes the change in momentum due to bulk motion (advection), while the right side describes the change in momentum due to the pressure gradient, Reynolds stress, and the body force. The $z$ direction velocities $w$ and $w^{\prime}$ are negligible, making the equation in the streamwise direction,

$$
\bar{u} \frac{\partial \bar{u}}{\partial x}+\bar{v} \frac{\partial \bar{u}}{\partial y}=-\frac{1}{\rho} \frac{\partial \bar{p}}{\partial x}-\frac{\partial}{\partial x}\left[\overline{u^{\prime} u^{\prime}}\right]-\frac{\partial}{\partial y}\left[\overline{u^{\prime} v^{\prime}}\right]-F_{x} .
$$

The energy equations are used to describe energy transport in the turbulent 
boundary layer. The general boundary layer energy transport equation is given by:

$$
\rho c_{p}\left(\overline{u_{j}} \frac{\partial \bar{\theta}}{\partial x_{j}}\right)=\frac{\partial}{\partial x_{j}}\left(k \frac{\partial \bar{\theta}}{\partial x_{j}}-\rho c_{p} \overline{u_{j}^{\prime} \theta^{\prime}}\right),
$$

where $\theta$ is the temperature, $c_{p}$ is the specific heat, and $k$ the thermal conductivity. Inner and outer equations are described by Wang and Castillo [39]. The infinite solar farm in this experiment lies in the inner region where the $x$ and $z$ heat flux terms are negligible in fully developed flow. Integrating along the remaining $y$ direction, the inner energy equation for a forced convection turbulent boundary layer is given as,

$$
-\frac{q_{w}^{\prime \prime}}{\rho c_{p}}=a \frac{\partial \bar{\theta}}{\partial y}-\overline{v^{\prime} \theta^{\prime}}
$$

where $q_{w}^{\prime \prime}$ is the heat flux wall-normal to $y$, and $a$ is the thermal diffusivity $k /\left(\rho c_{p}\right)$. The left side of the equation represents heat flux per volumetric heat capacity, the first term on the right side represents the conduction, and the second term on the right the turbulent heat flux. If conduction through air is minimal, the equation becomes:

$$
\frac{q_{w}^{\prime \prime}}{\rho c_{p}}=\overline{v^{\prime} \theta^{\prime}}
$$

Considering $\rho$ and $c_{p}$ as nearly constant in the temperature ranges considered, Eq. 2.14 demonstrates the proportionality of heat flux to the wall-normal velocity fluctuations. 


\section{Chapter 3}

\section{Experimental Methods}

\subsection{Experimental Facility}

Experiments were performed in the Portland State University wind tunnel using the system shown in Fig. 3.1. The closed-circuit wind tunnel consists of a $0.8 \mathrm{~m} \times$ $1.2 m$ cross section with a testing length of $5 m$, with a contraction ratio of 9:1 to facilitate low turbulence intensity. Tunnel working speeds range up to $30 \mathrm{~ms}^{-1}$. The tunnel walls were constructed of Schlieren-grade annealed float glass. The ceiling was inclined upwards along its length in order to ensure a zero pressure gradient within the test section.

Freestream turbulence intensity was controlled with a grid in the entrance to the test section, shown in Fig. 3.1. The grid system is comprised of $10 \mathrm{~cm}$ square winglets attached to 6 horizontal and 7 vertical rods, wherein each rod is independently activated [40]. In this study, active grid (AG) refers to these rods being actuated by motors, and passive grid $(P G)$ refers to each winglet held motionless and perpendicular to the incoming flow. Changing the rotation speed of the winglets allows the turbulence level in the boundary layer to be adjusted. Two active grid settings were used, designated $A G_{L o}$ and $A G_{H i} . A G_{L o}$ sent the motors a new rotation 


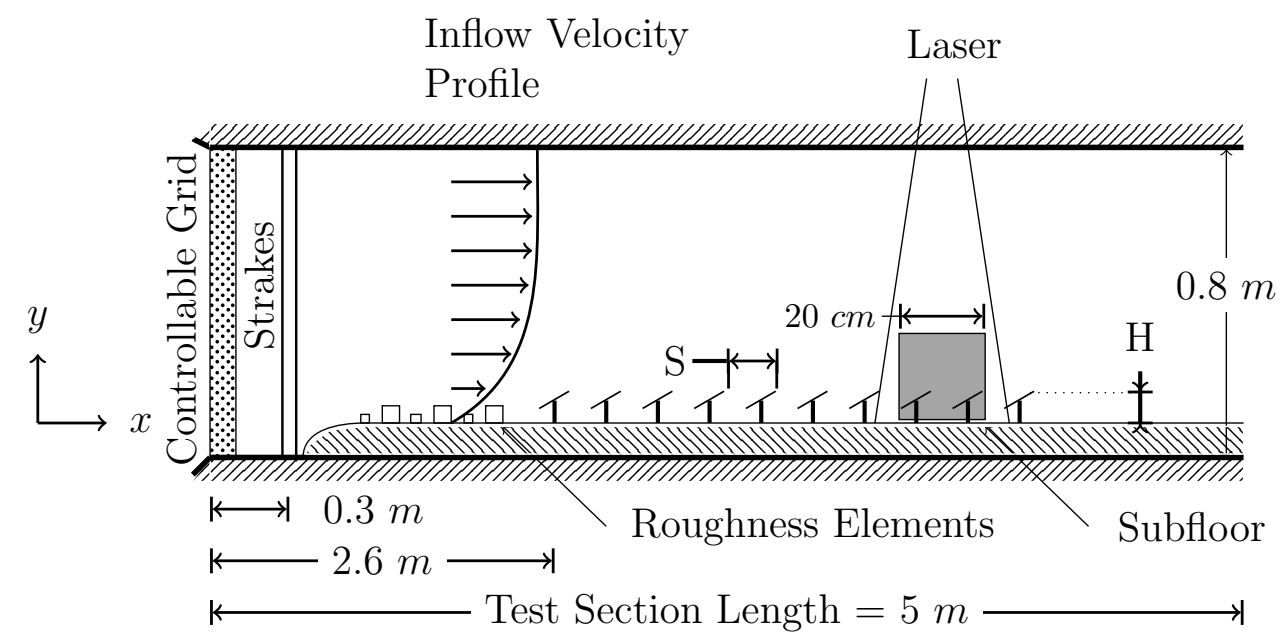

Figure 3.1: Schematic of wind tunnel experimental setup, side view. Flow is from left to right. Roughness elements shown as squares represent chains of two distinct sizes. Panels shown at angle of $+30^{\circ}$. Greyed square represents where images were captured using the PIV camera. Schematic not to scale.

speed every second in the range of $0.5-1.5 \mathrm{~Hz}$, and $A G_{H i}$ in the range 1.0-2.0 $\mathrm{Hz}$. In this experiment, the tunnel nominal freestream turbulence intensity with passive grid, roughness elements, and strakes was $4 \%$. The active grid with roughness elements and strakes gave TI values between $12-15 \%$.

Downstream of the active grid, shown in Fig. 3.1, the inflow was further conditioned with vertical strakes. Exact dimensions for the strakes are described further in Cal et al. [41] . Downstream of the vertical strakes, an acrylic floor standing 25 $m m$ above the original glass tunnel floor was used to allow wires to run underneath without disturbing the incoming flow. The transition to this raised floor was made using a quarter ellipsoid shape as shown in Fig. 3.1.

Above the subfloor and downstream of the active grid and strakes, a series of multi-scale roughness elements were used for further inflow conditioning as shown in Fig. 3.1. The roughness elements were two sizes of chains: chains (plastic, $25 \mathrm{~mm}$ 
wide $\times 50 \mathrm{~mm}$ long $\times 6 \mathrm{~mm}$ thick, 5 total) and chains (metal, $7 \mathrm{~mm}$ wide $\times 7 \mathrm{~mm}$ long $\times 1 \mathrm{~mm}$ thick, 30 total). The chains were spaced with $5 \mathrm{~cm}$ streamwise spacing, with every third chain being a larger chain, beginning $10 \mathrm{~cm}$ downstream of the strakes. The strakes and the roughness elements combined recreate an atmosphericlike boundary layer flow scaled with respect to the solar farm.

Using the log region of the measured inflow profile, the friction velocity $u_{\tau}$ and an effective roughness scale $y_{0}$ can be determined. The friction velocity $u_{\tau}$ and wall friction $\tau_{w}$ are defined as,

$$
u_{\tau}=\sqrt{\frac{\tau_{w}}{\rho}} \quad \tau_{w}=\left.\mu \frac{\partial U}{\partial y}\right|_{y=0}
$$

where $\mu$ is the dynamic viscosity. PIV measurements cannot be made sufficiently close to the tunnel floor so as to resolve the gradient of $U$ at the floor. Therefore, the effective friction velocity $\left(u_{*}\right)$ was obtained from a fit of the velocity profile shown in Fig. 3.2c, using the slope of the line $m$ in $u_{*}=m \kappa$, where $\kappa=0.4$ denotes the Von Kármán constant.

In order to characterize the flow in the wall-normal direction, mean vertical profiles of streamwise velocity and Reynolds stresses for the passive grid inflow case were analyzed in Fig. 3.2. The inflow data was fitted using the log law where the boundary layer is described as,

$$
U(y)=\frac{u_{*}}{\kappa} \ln \left(\frac{y}{y_{0}}\right)
$$

where $y$ denotes the wall-normal coordinate, and $y_{0}$ the roughness length. Fig. 3.2c 
shows the data fitted using the $\log$ law. The friction velocity $u_{*}$ calculated from the fitted line was $0.36 \mathrm{~ms}^{-1}$. The resulting roughness length for the passive grid case was $4.8 \mathrm{~mm}$; for the scaled atmospheric boundary layer this roughness length would scale to a distributed roughness height of $15.8 \mathrm{~cm}$, which indicates the boundary layer in the wind tunnel matches that of the ABL in 'open field' conditions, and is appropriate for PV solar farms in open terrain.

The mean velocity and Reynolds stress profiles resulting for the passive grid case are shown in Fig. 3.2. Spatial coordinates were normalized using $H$, the height of the top of the solar panel off of the tunnel floor. Normalized streamwise velocity is shown in Fig. 3.2a, while Fig. 3.2b shows the Reynolds shear stress profile. Near the wall, $\overline{u^{\prime} u^{\prime}} / U_{0}^{2}$ shows a peak, as would be expected as a boundary is approached. Both $\overline{v^{\prime} v^{\prime}} / U_{0}^{2}$ and $-\overline{u^{\prime} v^{\prime}} / U_{0}^{2}$ decrease near the wall, as the vertical motion is tempered at the wall boundary.

Additional inflow statistics were calculated for the active grid settings, $A G_{L o}$ and $A G_{H i}$. Roughness lengths of $y_{0}=1.0 \mathrm{~mm}$ and $y_{0}=1.1 \mathrm{~mm}$ corresponding to wind tunnel scale were calculated for the $A G_{L o}$ and $A G_{H i}$ cases respectively. Friction velocity resulting from the log-law fit was $u_{*}=0.26 \mathrm{~ms}^{-1}$ for both cases. The roughness length was approximately $20 \%$ of the calculated value for the passive grid case, and is still indicative of open terrain.

\subsection{Solar Farm Fabrication and Heating}

A solar farm was experimentally simulated using forty model panels. Each panel is comprised of four layers, each $254 \mathrm{~mm}$ wide $\times 50.8 \mathrm{~mm}$ long: 6061 aluminum flat bar (1.6 $\mathrm{mm}$ thick), Kapton heater (0.1 $\mathrm{mm}$ thick), aerogel insulation (2 $\mathrm{mm}$ 


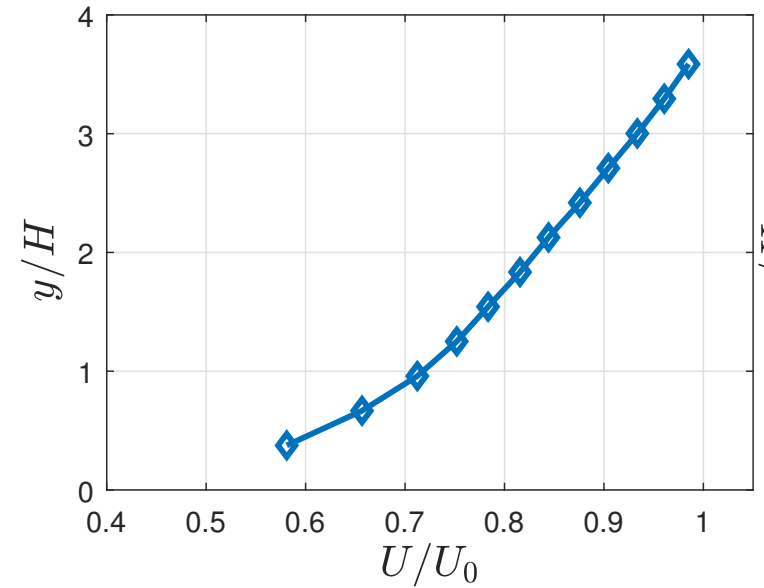

(a) Mean Inflow Velocity Profile

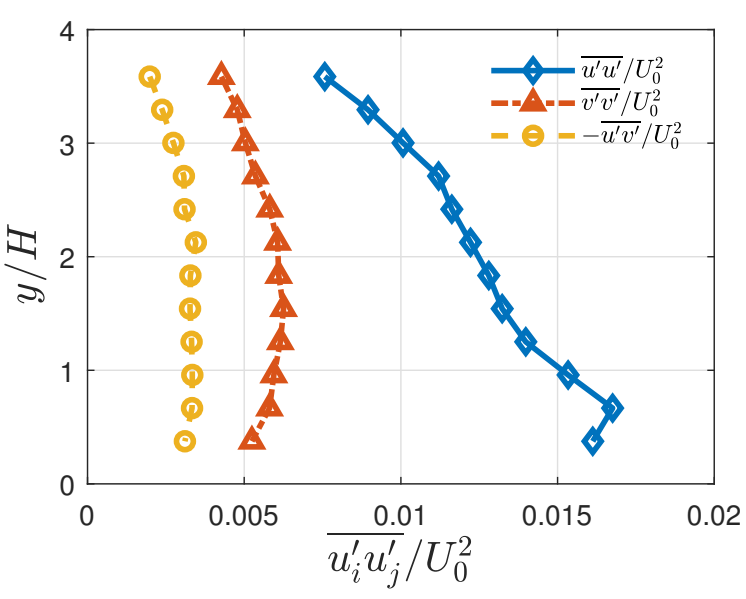

(b) Reynolds Normal and Shear Stresses

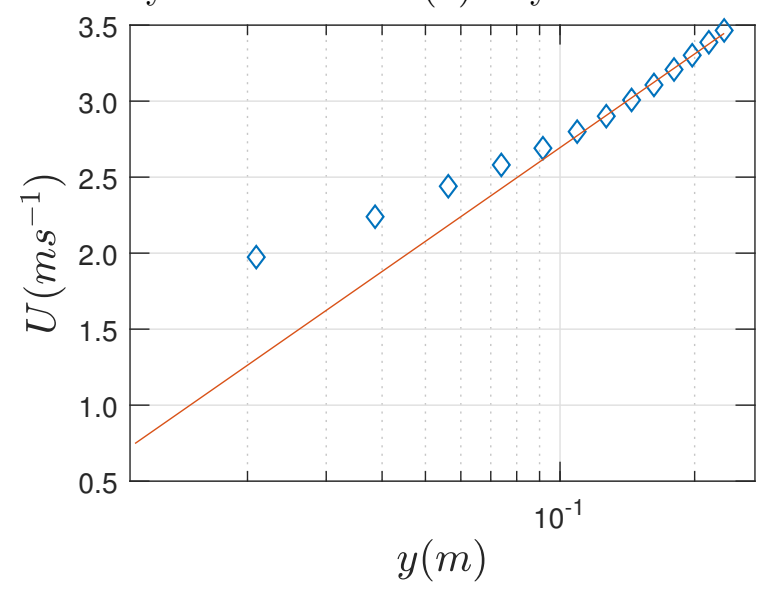

(c) Mean velocity profile in lin-log units

Figure 3.2: Inflow statistics, passive grid, in front of first row. The rough wall boundary layer profile is consistent with a wind tunnel scale roughness length of $y_{0}$ $=4.8 \mathrm{~mm}$ and $u_{*}=0.36 \mathrm{~ms}^{-1}$

thick), and 6061 aluminum flat bar (1.6 $\mathrm{mm}$ thick). The complete panel structure had a thickness of $5.3 \mathrm{~mm}$ and is shown in Figs. 3.3 and 3.4. Comparing with a full size solar farm with representative dimensions of $1.68 \mathrm{~m}$ long $\times 0.03 \mathrm{~m}$ thick placed side by side in long rows, these dimensions represent a 1:33 scale. The full scale module dimensions were pulled from the manufacturer SolarWorlds sunmodule, on 
their SW 254 mono version 2.0 panel. These panels were chosen for comparison due to their use at the Denver Federal Centers 3.2 $M W$ solar farm, where convection heat transfer data was taken for field comparison to future studies.

In the nominal case of $30^{\circ}$ inclination angle, the farm has a blockage ratio of $2.7 \%$. This low blockage ratio allowed the wake of the panels to develop freely upwards into upper portions of the tunnel so as to represent vertically unconstrained outdoor conditions.

A convergence study at $3.9 \mathrm{~ms}^{-1}, 30^{\circ}$ inclination, and $450 \mathrm{Wm}^{-2}$ demonstrated that the adiabatic heat transfer coefficient $h_{a d}$ reached a constant value after 6-7 panels, indicating fully developed flow. Ten rows of panels were used, 4 columns wide, in order to provide symmetry in the $z$ direction. All panels were mounted with a height of $B=38 \mathrm{~mm}$ between the tunnel floor and the bottom edge of panel as shown in Fig. 3.3a. This includes all cases where the panel inclination angle was changed, i.e. the panel angle pivots about the long edge lowest to the tunnel floor. In contrast, the $H$ dimension marks the top of the panels, which changes with varying inclination angles. Spacing between the rows of panels for all cases in this study was characterized as $S / B=2.3$, where $S$ is the distance from row edge to row edge $(88 \mathrm{~mm})$, shown in Fig. 3.1. For the nominal $30^{\circ}$ case, this spacing corresponds to where the ratio of streamwise distance between rows to length of the panel projected in the streamwise direction into the $x$ - $z$ plane is $1: 1$.

Each panel was uniformly heated using a commercially available electric resistance heater, a Watlow 120 Watt 120 Volt Kapton heater. Kapton heaters were chosen for three reasons: to reduce heater thickness and better mimic the aspect ratio of solar farms to the incoming wind velocity, for the low temperature coefficient 
of resistance of inconel $600\left(187 \cdot 10^{-6} \cdot \Omega \cdot \Omega^{-1} \cdot K^{-1}\right)$, and because the resistance of each heater creates an appropriate equivalent resistance when many panels are connected in parallel to a 48 Volt 6 A DC power supply. Each panel was sanded down with fine steel wool on both sides to ensure an even surface roughness profile, and wiped clean with isopropyl alcohol prior to heater installation. The panels were then painted matte black using an acrylic enamel spray paint. This paint improved reflection mitigation during use with the PIV as well as enabled the use of an IR camera for visual inspection of the setup.

Real solar panels operate commonly between $44^{\circ} \mathrm{C}$ and $65^{\circ} \mathrm{C}$ [3]. Surface temperatures observed in this study were between $30^{\circ} \mathrm{C}$ and $80^{\circ} \mathrm{C}$. Further, real solar

Thermocouple hole

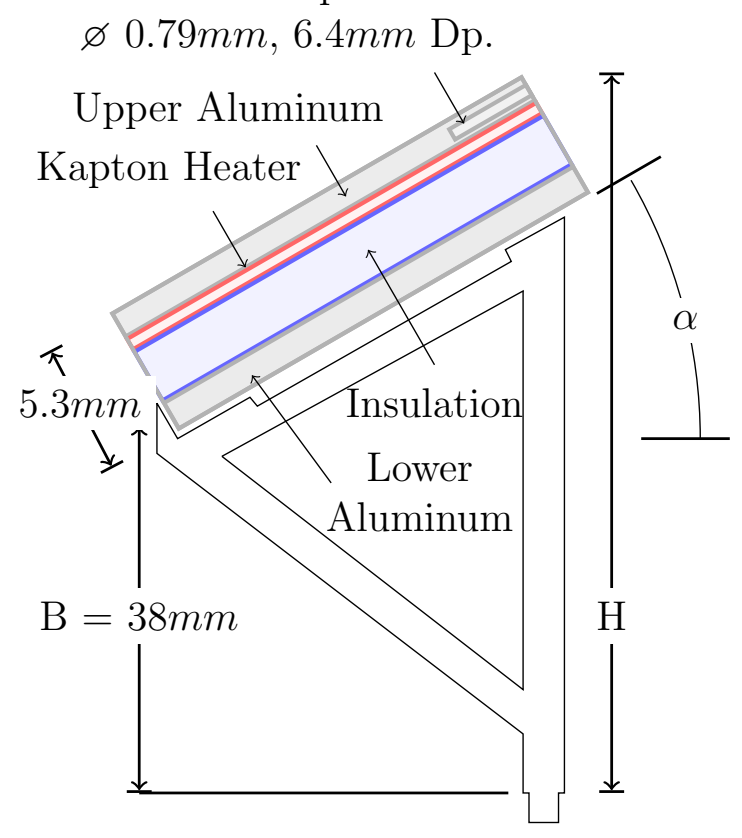

(a) Cross sectional panel view, cut through center hole. Panel thickness not to scale

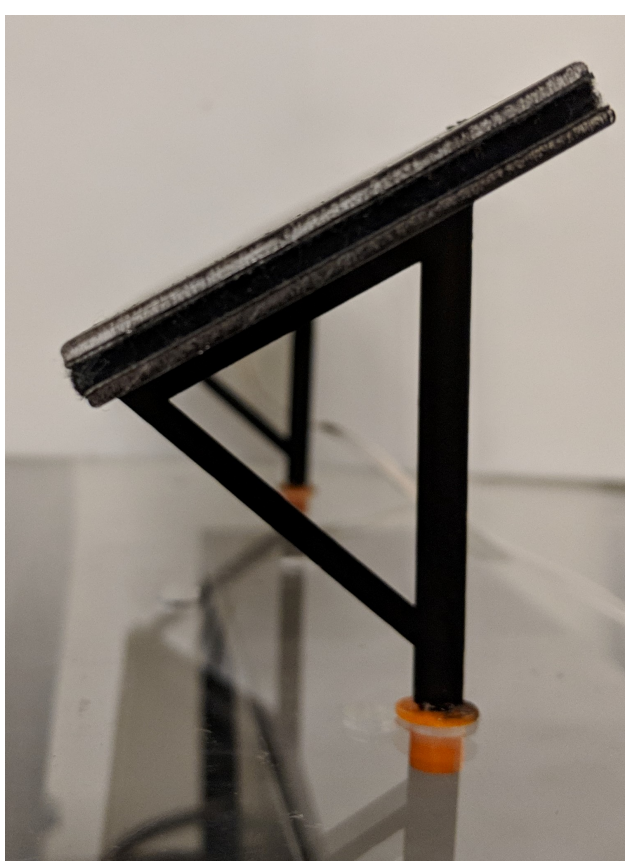

(b) Side view of model solar panel row

Figure 3.3: Schematic and photographic side views of model solar panels 
panels have upper and lower surface temperatures that are of the same order, usually with the lower surface measuring $3-5^{\circ} \mathrm{C}$ higher [19]. In this experiment, an insulating layer was used in order to increase the upper-lower surface temperature differential relative to the measurement uncertainty, thus reducing the overall $N u_{a d, u p}$ uncertainty.

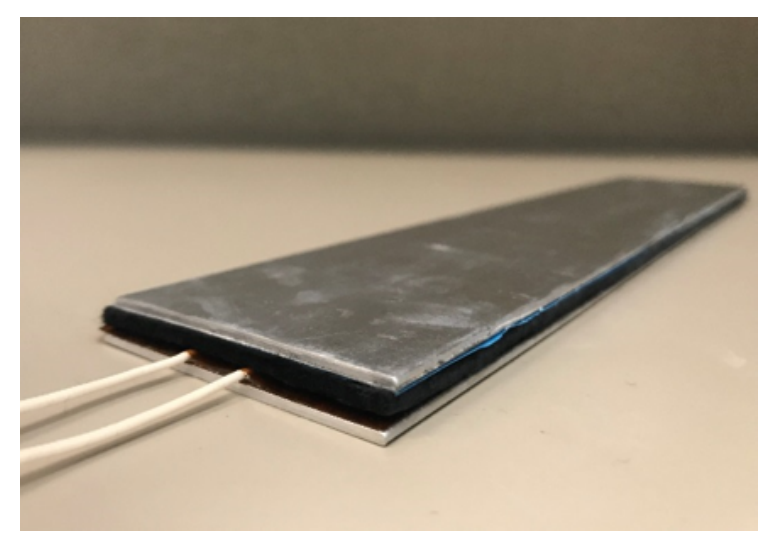

Figure 3.4: Individual Panel, prior to painting

Each heater was installed to the underside of the upper aluminum using the preapplied thermally conductive pressure sensitive adhesive to ensure proper thermal connection between the heater and mating Kapton heater. Each heater was firmly rolled on so as to avoid air pockets. The insulation and lower aluminum surfaces were held together with thermally conductive double sided CPU (computer processing unit) tape. Lead wires for power wires and thermocouples were routed along the panel edges so as to keep the panel surface clear, then routed down directly beneath the floor to avoid interfering with incoming flow. Each panel was held at the prescribed height and angle using two acrylic stands, spaced $153 \mathrm{~mm}$ on center as shown in Fig. 3.5.

Electric power was supplied to the panels by two $48 \mathrm{VDC} 6 \mathrm{~A}$ power supplies, 

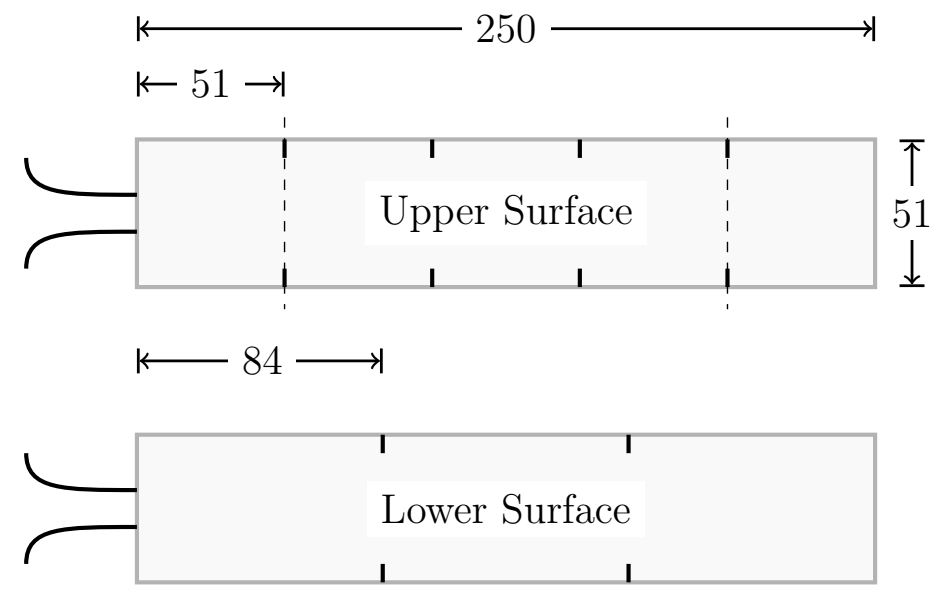

Figure 3.5: Model solar panel planform views. Thermocouple placement and support stand location (dashed lines) (units in $\mathrm{mm}$ )

with each power supply separately supplying twenty of the panels with power in parallel. The voltage drop across each panel measured with a digital multimeter on the Hewlett Packard (HP) data system described below. The voltage supplied to the heaters was maintained to within $19 \mathrm{mV}$. Ten panels were wired through switches which allowed for switching on and off, so that the adiabatic (unheated) temperatures could be quickly and reliably recorded.

To measure the surface temperatures, Type $\mathrm{T}$ Copper constantan $\left( \pm 0.5^{\circ} \mathrm{C}\right)$ thermocouples were placed underneath the top surface in a cavity $\varnothing 0.79 \mathrm{~mm}, 6.4$ $\mathrm{mm}$ deep, where the center of the cavity was located $0.635 \mathrm{~mm}$ from the panel surface. Each cavity was filled with a thermally conductive silicone (Halnziue silicone heatsink plaster, HY910) and a thermocouple inserted to full depth. The thermocouple junction itself was kept to a length of $2 \mathrm{~mm}$ or less. Fig. 3.3a shows a cross sectional view of a panel, with the scale inconsistent for visual clarity. All thermocouple lead wires were routed from their starting point along the edge of the module and covered with an anti-reflective gaffer tape. 
Ten out of the forty panels were instrumented with thermocouples. Of these, one contained twelve thermocouples (eight in upper surface, four in lower surface) and the other nine panels contained two each (one in upper surface, one in lower). The panel containing twelve thermocouples was constructed to better characterize the temperature distribution over a single panel, and the locations of thermocouples on that panel are shown in Fig. 3.5.

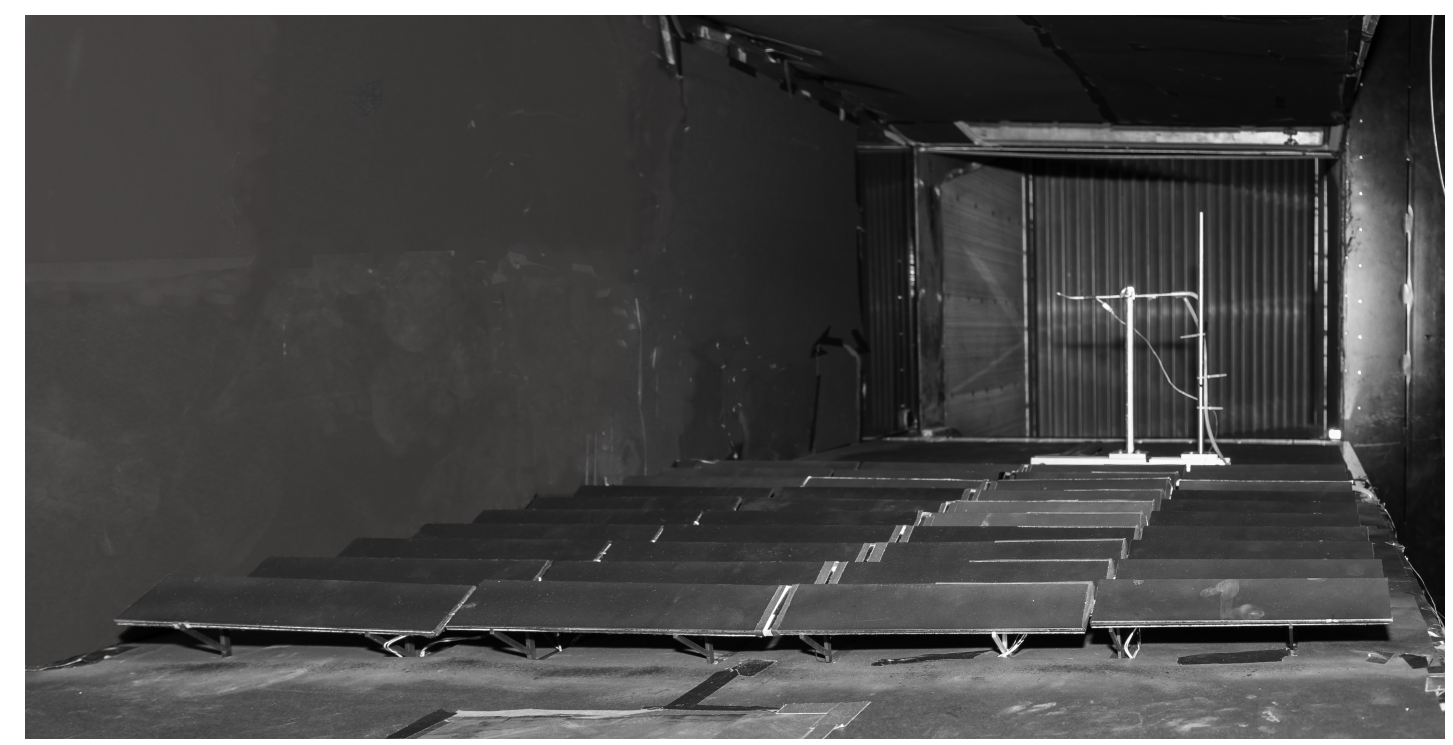

Figure 3.6: Model solar farm in experimental wind tunnel, viewed from upstream. Pitot tube shown in rear

It should be noted that the resulting temperature distribution indicates an imperfect boundary condition - it is neither perfectly isothermal or constant heat flux, thus indicating that one should generalize the results with caution. It is intended to approximate an isothermal boundary condition on the upper surface. However, a balance is struck in maintaining a realistic aspect ratio (i.e. not making the aluminum plate too thick) in order to keep the hydrodynamic flow similar. 


\subsection{Uncertainty Analysis}

Uncertainties in the convective heat transfer coefficient $h_{m}$ were estimated using the sequential pertubation technique outlined by Figliola and Beasley [42]. This approach and equations are outlined in Appendix B. Uncertainties arising from temperature measurement $\left( \pm 0.58^{\circ} \mathrm{C}\right)$, voltage $( \pm 0.072 \mathrm{VAC})$, current $\left( \pm 1.2 \times 10^{-6} \mathrm{~A}\right)$, spatial and temporal variations, and thermophysical properties were considered. Resulting uncertainty ranged from $8-20 \%$ depending on the case in question.

\subsection{Data Measurement and Acquisition}

\subsubsection{Temperature Measurement and Acquisition}

All temperature measurements were taken using Type T Copper Constantan $( \pm$ $0.5^{\circ} \mathrm{C}$ ) thermocouples (OMEGA TT-T-30-SLE), and recorded using an HP34970A data acquisition unit. Data was recorded at a frequency of $0.2 \mathrm{~Hz}$, and averaged over 5 minute periods after the change in surface temperature varied less than $0.08^{\circ} \mathrm{C}$ over a 5 min period. A single ice-point reference was used for the thermocouples. Each thermocouple was routed through a zone box to ensure reference junction temperature uniformity. Power levels used were chosen to ensure that the minimum incoming flow to heater temperature difference is greater than $7^{\circ} \mathrm{C}$. The maximum temperature difference was $20^{\circ} \mathrm{C}$.

All heater resistance values were calculated using the HP unit. Calculations were made at room temperature, using two wire measurements. Offsets were calculated for each lead wire resistance and used to correct the measured resistance values. Resistance for all resistive heaters was measured to within $0.012 \Omega$. 


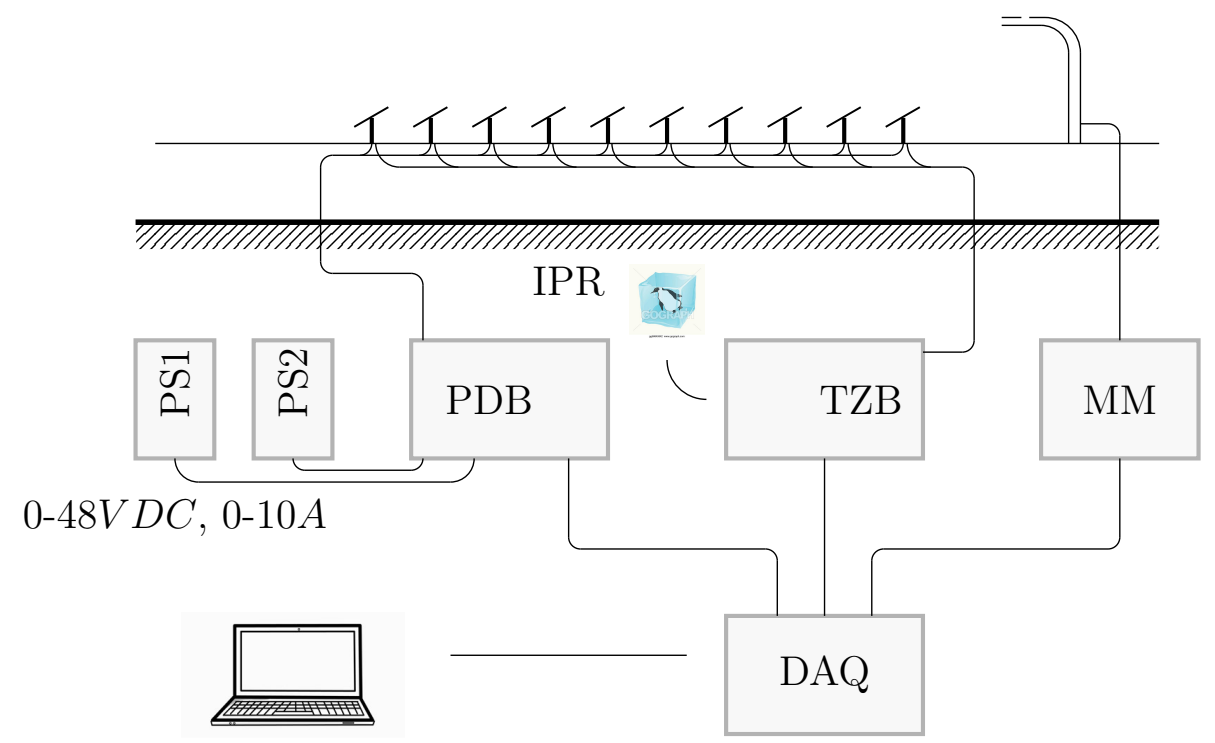

Figure 3.7: Data Acquisition Setup (PS1: Power Supply 1, PS2: Power Supply 2, PDB: Power Distribution Box, IPR: Ice Point Reference, TZB: Thermocouple Zone Box, MM: Micromanometer, DAQ: Data Acquisition unit

Spatial temperature variance on the upper surface of the panel was recorded throughout all experiments and included in the measurement uncertainty. Thermocouple measurements indicated that the entire surface of the panel was within $2^{\circ} \mathrm{C}$ to the measured reference probe at the center of each panel across the range of temperatures.

A Furness Controls FCO65 $200 \mathrm{~mm}$ pitot tube alongside a FCO510 micromanometer was used to measure the freestream velocity $0.4 \mathrm{~m}$ above the test section floor at the location of the last row of panels .

\subsubsection{Particle Image Velocimetry}

PIV measurements were obtained between the 8th and 9th row, such that the camera could capture the flow over the surface of the 9th panel. A LaVision PIV system was 
used, with an Nd:Yag (532 $\mathrm{nm}, 1200 \mathrm{~mJ}, 4 \mathrm{~ns}$ duration) double-pulsed laser and one 4MP ImagerProX charge-coupled device (CCD) cameras. Neutrally buoyant fluid particles (diethylhexl sebacate) were seeded into the flow upstream. In order to capture a plane in the center line of the tunnel, a single camera was aimed between rows, capturing flow in the $x-y$ plane (see Fig. 3.8).

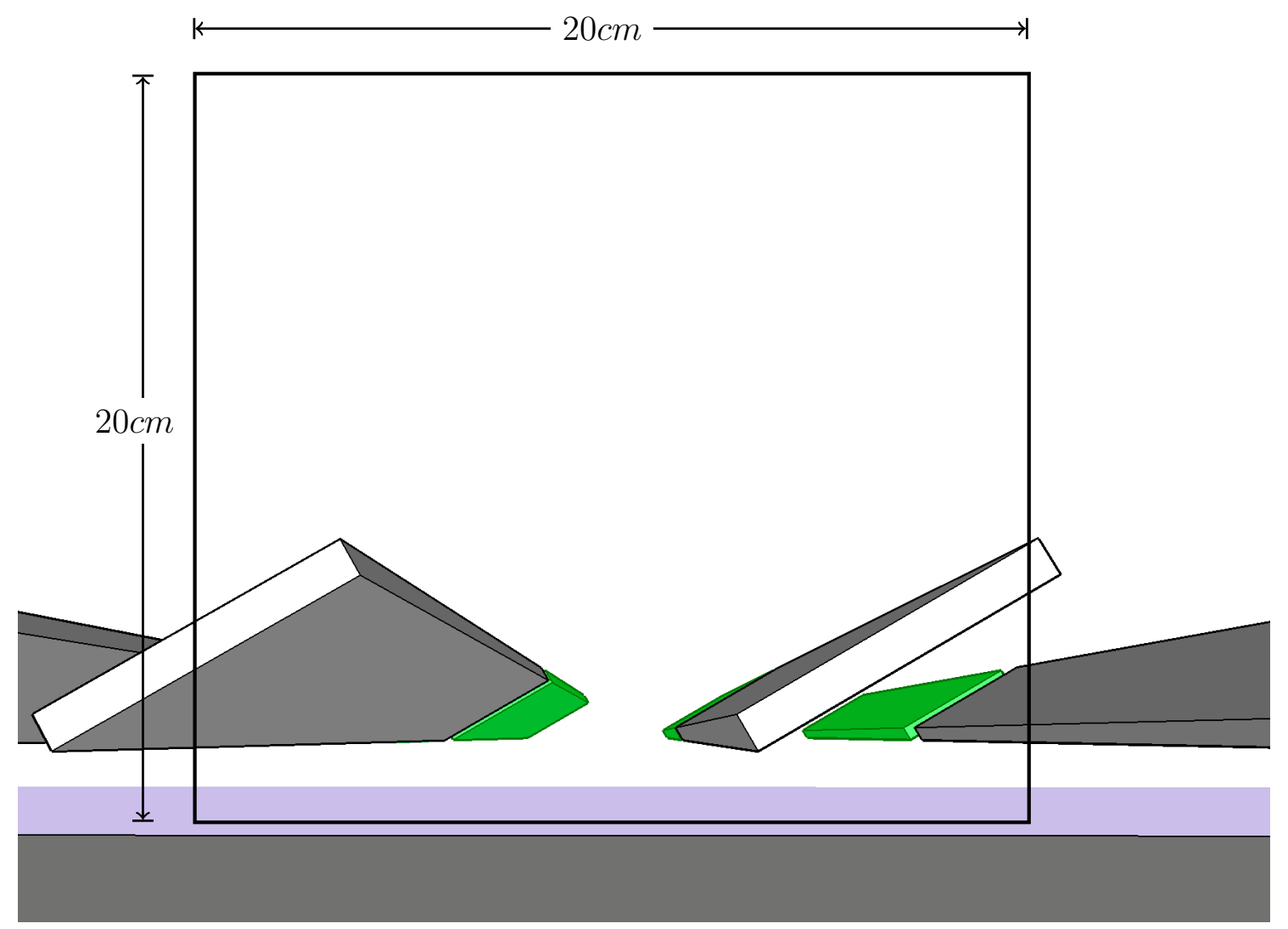

Figure 3.8: Solar Farm shown from PIV camera, perspective view. Columns of panels past PIV laser plane shown in green. Panels shown without stands.

The field of view was approximately $0.2 m \times 0.2 \mathrm{~m}$ with a vector resolution of $1.5 \mathrm{~mm}$. Cameras were calibrated before data was taken on a given plane using a standard two plane measurement plate. The laser plane thickness was approximately $1 \mathrm{~mm} .3000$ PIV image sets were collected for all cases and a convergence test was 
run calculating ensemble averages of 500, 1000, and 3000 samples. In order to reduce the reflectance for better PIV measurements, a $8 \mathrm{~mm} \times 50 \mathrm{~mm} \times 6 \mathrm{~mm}$ piece of transparent acrylic was placed between adjacent panels as shown in Fig. 3.6. This allowed the laser to pass through the surface instead of reflecting and interfering with camera measurements. The piece was cut so that the bottom corner was parallel to the floor to reflect light passing through the acrylic downwards, and is responsible for the narrow band of noise visible in Fig. 3.9 at $x \approx 40 \mathrm{~mm}$ below the panels.

The vector fields were calculated from raw images using a multi-pass Fast Fourier Transform (FFT) based correlation algorithm. The algorithm used reducing size interrogation windows: twice at $64 \times 64$ and twice at $32 \times 32$ pixels with $50 \%$ overlap. A resulting contour plot is shown in Fig. 3.9. Black lines signify the physical location of the panels, and white regions signify obstruction of camera view by the panels. An analysis was performed comparing the velocity and stress profiles resulting from three $x$-direction spatial averaging windows. Spatial averaging allows
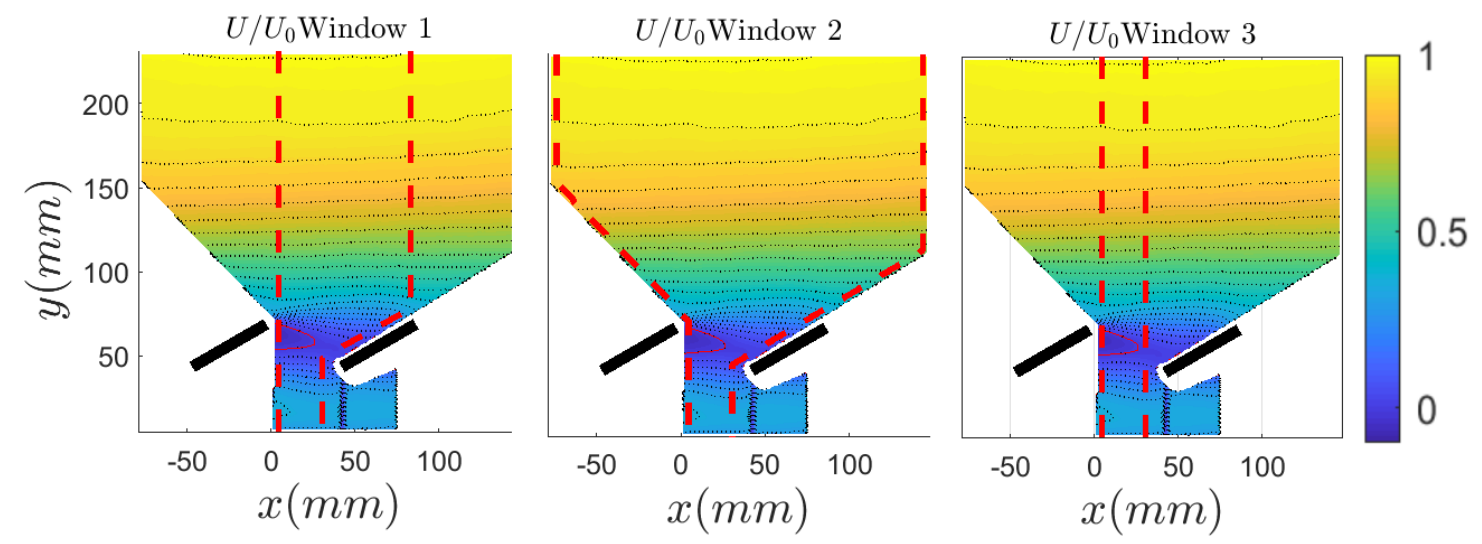

Figure 3.9: Velocity spatial contour plot resulting from PIV. Red lines denote region for $x$ direction averaging for velocity plots. 
for a comparison of key data from different cases. The three different regions for spatial averaging are designated by the red dashed lines in Fig. 3.9, designated Windows 1,2 and 3. The first window represents averaging over exactly one row, from the beginning of one panel to the beginning of the subsequent panel. The second window uses all available data, and the third window averages a section of uniform width running the entire height.

Fig. 3.10 shows the results for the mean streamwise velocity profiles obtained using the three averaging windows shown in Fig. 3.9. A slight change between the averaging windows of less than $2 \%$ of the maximum velocity is observed at a height of $y / H=1$, but otherwise $U / U_{0}$ shows relative insensitivity to the different averaging windows. Figs. 3.11 - 3.12 show the effect of varying spatial averaging windows on the wall-normal velocity and Reynolds stress profiles. Beginning with the vertical velocity contour shown in Fig. 3.11a, coherent structures are observed that vary along the $x$-direction, causing variations in the resulting wall-normal profiles. In the panel wake (near $y / H=0.8$ ) and just above the panel (near $y / H=1.2$ ), window

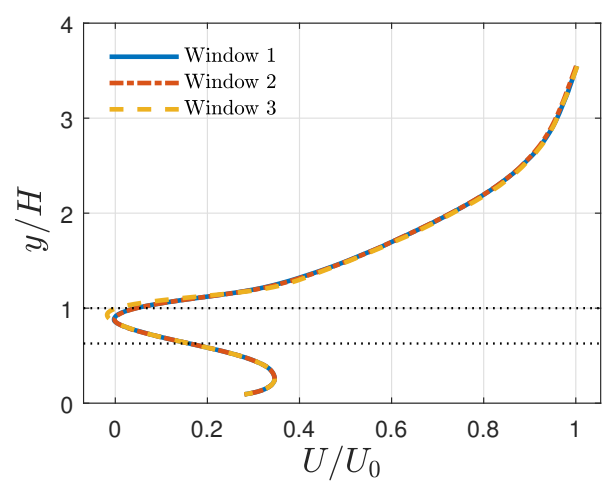

Figure 3.10: Streamwise-averaged profiles of normalized streamwise velocity for three different spatial averaging windows. 


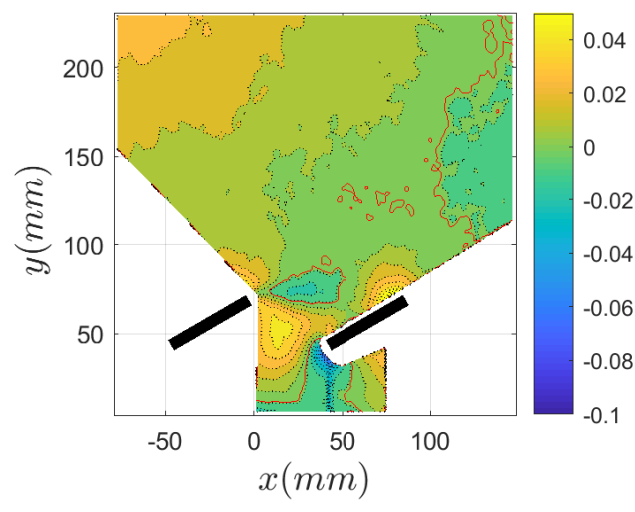

(a) $V / U_{0}$ : Vertical velocity contour

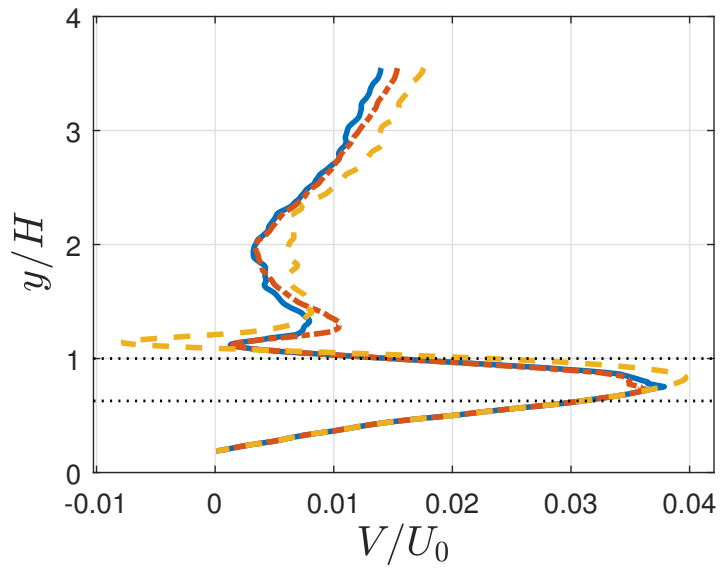

(b) $V / U_{0}$ : Vertical mean velocity profile

Figure 3.11: Streamwise-averaged profiles of normalized vertical velocity profiles calculated with different averaging windows

3 shows extremes $16 \%$ and $8 \%$ respectively greater than windows 1 and 2 . This is caused by the downward structure just above the panels at $x=10-50 \mathrm{~mm}$, and the upward structure near $x=75 \mathrm{~mm}$. The upper region, $y / H<1.5$, shows a increase of $4 \%$ from windows 2 and 3 to window 1 . The narrower averaging region in window 1 captures the downward velocity in between the panels but not the upwards velocity above the panels. This indicates that in interpretation of results for $V / V_{0}$, the magnitude of peaks observed near the top of the panels must be compared with caution. Overall trends remain the same between all three averaging regions.

Fig. 3.12 show the effect of varying spatial averaging windows on the Reynolds shear stress profile. Good agreement is observed between the 3 windows, with window 3 showing $3 \%$ larger extreme values near the top of the panel. These elevated values are due to the higher values of $-\overline{u^{\prime} v^{\prime}} / U_{0}^{2}$ near $x=40 \mathrm{~mm}$, which then decrease along the positive $x$-direction. Reynolds shear stress profiles for the three averaging windows show deviations similar in magnitude to the Reynolds normal stress 


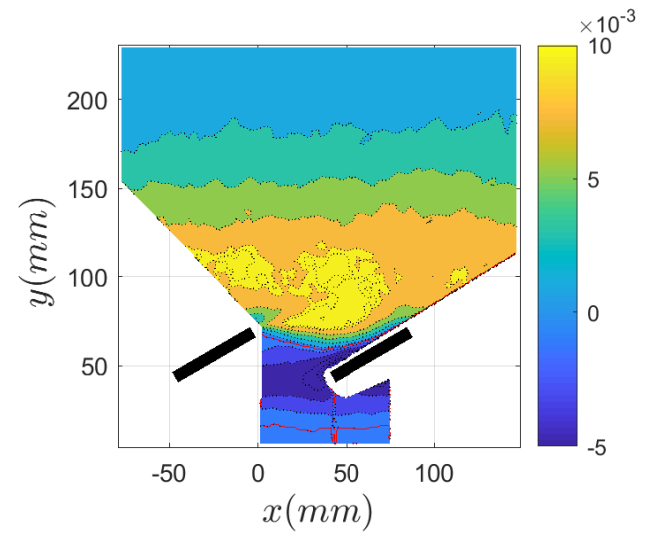

(a) $-\overline{u^{\prime} v^{\prime}} / U_{0}^{2}$ : Reynolds shear stress contour

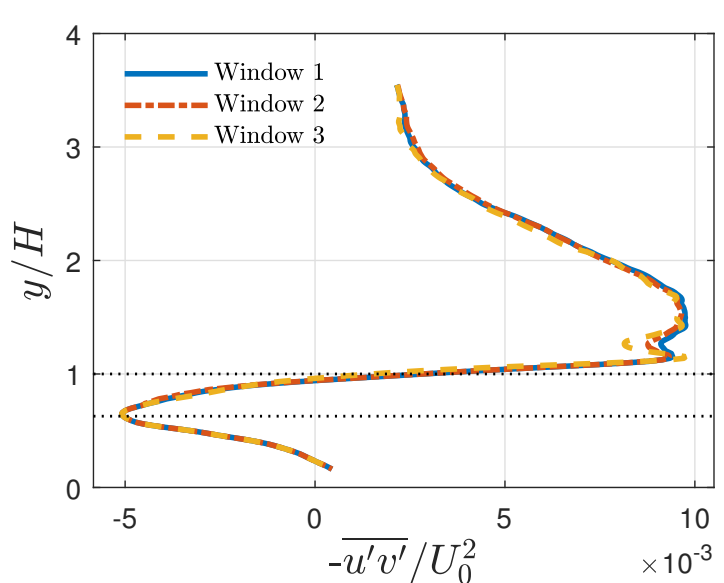

(b) $-\overline{u^{\prime} v^{\prime}} / U_{0}^{2}$ : Reynolds shear stress mean wall-normal profile

Figure 3.12: Streamwise-averaged profiles of normalized Reynolds shear stress profiles calculated with different averaging windows

deviations, which are thus not shown here.

Across all measured values, the maximum variation using the different averaging windows was $18 \%$, and throughout the majority of the wall-normal distance the values were the same. Spatial averaging in the $x$-direction was determined to be a valid approach to characterizing the behavior of the flow, since most of the key structures and flow changes exist in the wall-normal direction and that the averaging windows are in good agreement. For all streamwise-averaged profiles shown in this study, Window 1 was chosen as it represents an average over exactly one row, thus being representative of an infinite solar farm.

\subsection{Test Matrix}

Table 3.1 shows the values for which each parameter was varied. Nominal values are listed in the top row. Velocity measurements ranged from 1 to $5.7 \mathrm{~ms}^{-1}$. 


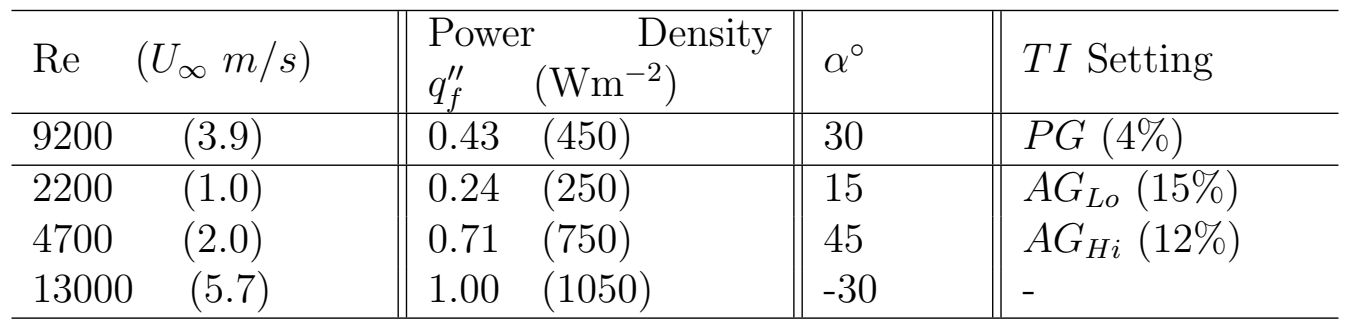

Table 3.1: Test matrix: parameter values used in experiments

Power density ranges from 250-1050 $\mathrm{Wm}^{-2}$ and were normalized with the nominal value for solar direct radiation $q_{0}^{\prime \prime}=1050 \mathrm{Wm}^{-2}$, giving $q_{f}^{\prime \prime}=q_{e}^{\prime \prime} / q_{0}^{\prime \prime}$. A typical solar panel efficiency is $15 \%$, and solar irradiation ranges from 0 to $1100 \mathrm{Wm}^{-2}$. Power levels were controlled to within $5 \mathrm{Wm}^{-2}$ of the target values. The nominal angle used for the experiment, $30^{\circ}$ from the horizontal, represents an optimum angle for the Southwestern United States [43]. A total of 16 cases were run for Reynolds number and power density (4 power levels at each Reynolds number), and 16 total were run for the 4 angles ( 1 power level, $q_{0}^{\prime \prime}=0.42$, and 4 Reynolds numbers at each angle).

A comparison of the Reynolds number between a full scale farm and the experimental setup shows that it scales approximately equal to the size ratio, as the inflow velocities are comparable to those prevalent in field conditions. Hence, dynamic similarity on the individual panel level will certainly not be achieved. However, the focus of this study is on the large-scale transport properties of the turbulence, where Reynolds number effects are known to be less dominant. After the first panel, the array induced turbulence should make the Reynolds numbers seen in the array much closer to full scale farms.

The blockage ratio of the solar farm model to the wind tunnel area was calculated 
using the projected area of the panels onto a plane perpendicular to the incoming flow direction and the cross sectional area of the wind tunnel. This ratio, in this experiment holding a value of $1: 41$, is a relatively low value, allowing for mostly unimpeded expansion of the wakes. 


\section{Chapter 4}

\section{Results}

Results presented here assess the impact of Reynolds number, turbulence intensity, and panel inclination angle for both the thermal and the flow field characteristics in an infinite PV solar farm. To characterize the flow field mean statistics for streamwise and vertical velocity, the Reynolds normal stresses and the Reynolds shear stresses are shown for each parameter variation. For thermal characterization, both Nusselt number and surface temperature are used. The heat transfer and flow field statistics together present a picture of the flow through the solar farm and the resulting effect on temperature. This in turn provides the foundation necessary to enhance the flow and reduce the temperature in a solar farm.

Nominal conditions referenced here correspond to the top row of Table 3.1: Re= 9200, $q_{f}^{\prime \prime}=0.43, \alpha=30^{\circ}$, and passive grid. Unless otherwise stated, all thermal results are for the panel located in row 8, column 3 of 4 (centered at $z=0.13 m$, where $z=0 m$ is at the centerline of the tunnel). Mean velocities and Reynolds stresses are normalized in the wall-normal direction using the height of the top of the panel from the ground $(H)$. Two spatial regions are classified for discussion as follows: underneath the panels is "sub-panel flow" $(0<y / H<0.7)$, and space between panels directly downstream of a panel as the "direct wake" $(0.7<y / H<1.1)$. 


\subsection{Reynolds Number and Power Density Effects}

The variation of Nusselt number with Reynolds number is shown in Fig. 4.1 for four input power levels $\left(q_{f}^{\prime \prime}=0.24,0.42,0.71,0.99\right)$. Trends for the upper surface and lower surface are represented with solid and dashed lines respectively. Each data point reported at $q_{f}^{\prime \prime}=0.24$, and $q_{f}^{\prime \prime}=0.42$ is an average from three individual measurements. Run-to-run variability is demonstrated to be less than $4 \%$; consequently, the $q_{f}^{\prime \prime}=0.71$ and $q_{f}^{\prime \prime}=0.99$ cases were determined from single measurements.

For each case, a strong dependence of $N u_{a d, u p}$ and $N u_{a d, l o}$ on Reynolds number

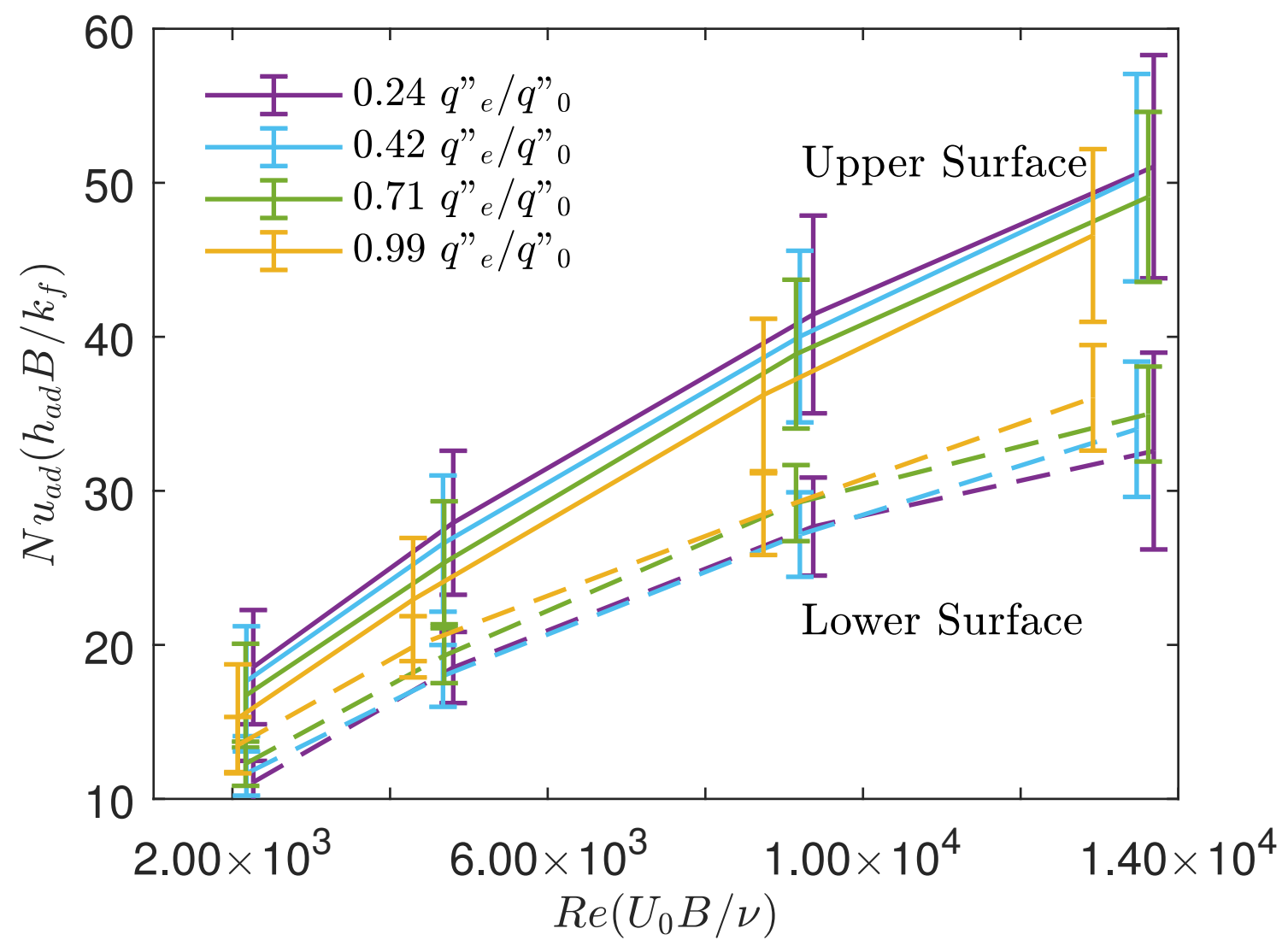

Figure 4.1: Adiabatic heat transfer for upper and lower surfaces of Row 8 
is observed. $N u_{a d, u p}$ is the ratio of convection to conduction heat transfer from the upper panel surface, defined in Eq. 2.7. For the nominal case, $N u_{a d, u p}$ increases by a factor of 2.9 from 17.6 to 50.3 from the lowest Reynolds number to the highest, 2200 to 13500. Similar trends are observed for $N u_{a d, u p}$ at the three remaining tested power levels. The increase in $N u_{a d, u p}$ with increasing $R e$ is due to the increase in convection efficiency at higher wind speeds. Likewise for the lower surface, $N u_{a d, l o}$ for the nominal case begins at 11.7 and increases 2.9 times from the lowest to the highest Reynolds number.

For both the upper and lower surface, there is an apparent reduction in dependence on Reynolds number as the Reynolds number increases: at lower Reynolds number, Nusselt number is more sensitive to changes in Reynolds number. This suggests that a change in the wake structure occurs, where the wake recovery varies between the lower and higher Reynolds numbers.

As input power level increases, $N u_{a d, u p}$ decreases slightly across all Reynolds numbers by a mean of $9 \%$ between the lowest and highest power level cases. Compared with $N u_{a d, u p}$, for the lower surface a reverse trend (increasing $N u_{a d, l o}$ ) is observed as power level increases. Nusselt number is normalized with respect to temperature, and thus the observed variations in Nusselt number as power input changes in Fig. 4.1 must be accounted for. Two explanations are proposed for the observed behavior: 1) The flow itself changes due to the effect of buoyancy or 2) increased parasitic losses. The latter explanation is suspected, as conductive loses down the heating wires would lower the temperatures of the upper surface due to the Kapton heater and its lead wires being physically attached to the underside of this surface. Radiative losses are accounted for as shown in Fig. 2.1, suggesting that 
these conductive losses could account for the reduction. These lower temperatures on the upper surface would cause the system shown in Fig. 2.2 to interpret more heat going to the rear surface. This would cause an apparent decrease in $N u_{a d, u p}$ on the upper surface and an apparent increase in $N u_{a d, u p}$ on the lower surface, both of which are observed in Fig. 4.1.

Fig. 4.2 shows a comparison of the present study nominal case with other studies exploring the relationship between Reynolds number and Nusselt number over flat surfaces. Two studies selected are wind tunnel experiments over arrays of heated rectangular blocks, and two are the classical Nusselt number relations for flow over an isothermal plate [44]. Across the selected studies, magnitudes vary by a factor of 2, with the Sparrow study showing the maximum Nusselt numbers. However, the characteristic length used in that study is the channel height, or $8 / 3$ the height of the objects off the floor (B), and when accounting for this the Sparrow study would actually show lower Nusselt numbers than the present study, this time lower by $50 \%$ [13]. The Arvizu experiment used the height of the heated blocks off of the tunnel floor as the characteristic length, which is more comparable to the length chosen in this study.

The present study is in good agreement with the studies shown. Lower values (Sparrow, length scale adjusted) or almost equivalent (Arvizu) were shown for the experimental results. Several factors may bend the comparisons in each direction. Lower channel heights $(H)$ increase the blockage ratio, increasing the $N u_{a d, u p}$ in relation to this study. On the other hand, the blocks are protruding from the floor and no flow is allowed underneath, thus decreasing the flow near the panels. Similar trends are observed for each, where the effect of Reynolds number begins to weaken 


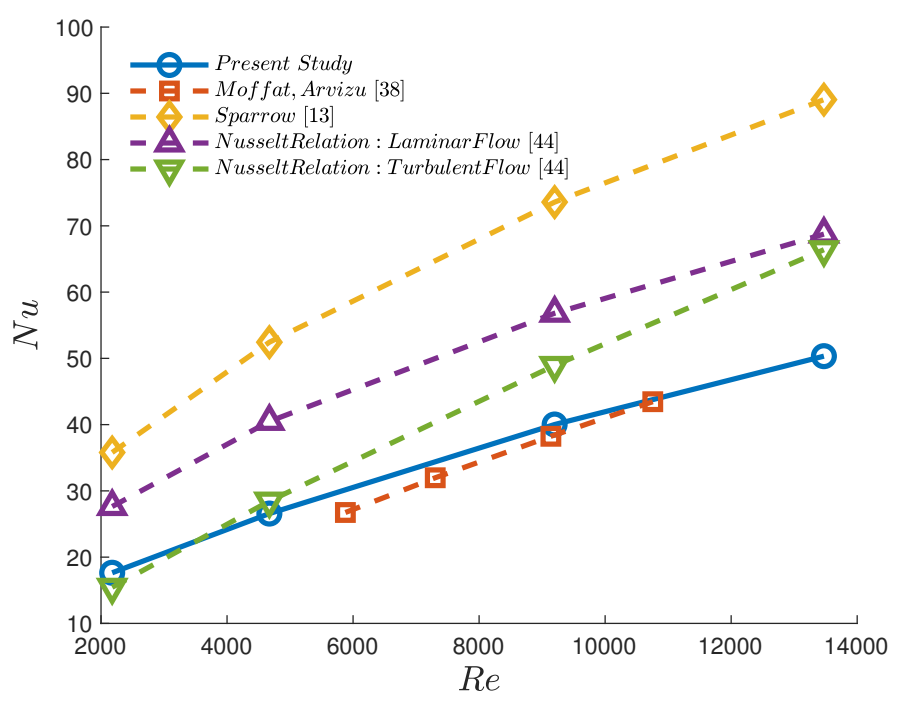

Figure 4.2: Comparison of Nusselt number to literature

as it reaches higher and higher values.

\begin{tabular}{llll}
\hline Author & Equation & $\begin{array}{l}\text { Char. } \\
\text { Length }\end{array}$ & Comment \\
\hline \hline Moffat [38] & $N u_{B}=C R e_{B}^{0.075}$ & $\mathrm{~B}$ & $\begin{array}{l}\text { Heated rectan- } \\
\text { gular blocks }\end{array}$ \\
\hline Sparrow [13] & $\overline{N u}=0.86 \operatorname{Re}^{1 / 2} \operatorname{Pr}^{1 / 3}$ & $\frac{8}{3} B$ & $\begin{array}{l}\text { Heated rectan- } \\
\text { gular blocks }\end{array}$ \\
\hline $\begin{array}{l}\text { Nusselt Relation } \\
{[44]}\end{array}$ & $\overline{N u}=0.664 \operatorname{Re}^{1 / 2} \operatorname{Pr}^{1 / 3}$ & $\mathrm{~L}$ & Laminar \\
\hline $\begin{array}{l}\text { Nusselt Relation } \\
{[44]}\end{array}$ & $\overline{N u}=0.037 \operatorname{Re}^{4 / 5} \operatorname{Pr}^{1 / 3}$ & $\mathrm{~L}$ & Turbulent \\
\hline
\end{tabular}

Table 4.1: Nusselt Number correlation equations 


\subsubsection{Flow field statistics}

PIV measurements of the normalized streamwise and wall-normal mean velocity profiles and turbulence statistics are presented in Fig. 4.3 and Fig. 4.4. Data was taken at four Reynolds numbers. The velocity and turbulence statistics results were normalized by the mean freestream speed $U_{0}$ (or $U_{0}^{2}$ for Reynolds stress components $\overline{u^{\prime} u^{\prime}}, \overline{v^{\prime} v^{\prime}}$ and $\left.-\overline{u^{\prime} v^{\prime}}\right)$ as measured at the top of the PIV viewing window. Each figure contains black horizontal dotted lines designating the top and bottom of the panel inclined at $30^{\circ}$.

Fig. 4.3a shows the normalized streamwise mean velocity profile as a function of normalized wall-normal distance at four Reynolds numbers. For the nominal case, $U / U_{0}$ shows a local maximum at $y / H=0.3$ in the sub-panel flow, and shows the largest velocity deficit at $y / H=1$. Above the panels at $y / H>1$, the streamwise velocity $U / U_{0}$ increases with height, indicating the boundary layer growth above the panels. This boundary layer was analyzed for its velocity characteristics, which demonstrated that the velocity assumes a logarithmic profile in the vertical range $1.3<y / H<2.9$.

Due to the significant differences in length scale dynamic similarity was not achieved in this study, similar to other wind tunnel turbulence studies [41]. However, these results show that the flow is relatively Reynolds number independent especially at $R e>4700$ where the effects of Reynolds number become small. The only variation is observed at the lowest Reynolds number $R e=2200$, where a $6 \%$ increase over nominal is observed at the local maxima at $y / H=0.3$ and a $3 \%$ decrease between $3<y / H<4$. This Reynolds number independence indicates that the results can still apply to full scale solar farms. 
In Fig. 4.3b, normalized wall-normal mean velocity profiles $V / U_{0}$ are shown as a function of normalized wall-normal coordinates at four Reynolds numbers. Across all heights $y / H$, the $V / U_{0}$ is generally positive, due to the inclined panels directing incoming flow upwards. There is a substantial positive gradient in the wake of the panels, increasing from the floor to $y / H=0.95$ and decreasing at heights just above the top of the panels. In the region $1.1<y / H<1.4, V / U_{0}$ is slightly negative, indicating a recirculation caused by shedding off of the blunt upper edge of the panel and as expected by wake recovery. Continuity dictates that a positive gradient of $U$ in $x$ (i.e. wake recovery) requires a negative gradient of $V$ in $y$, as observed in the profile. Across the wall-normal coordinates, the magnitude of $V / U_{0}$ is generally much lower than the streamwise velocity; the maximum $V / U_{0}$ is only $4 \%$ maximum $U / U_{0}$

Above the panels at $y / H>1.2$, the $V / U_{0}$ appears to be Reynolds number dependent; higher $V / U_{0}$ values are observed at lower Reynolds numbers. The variation

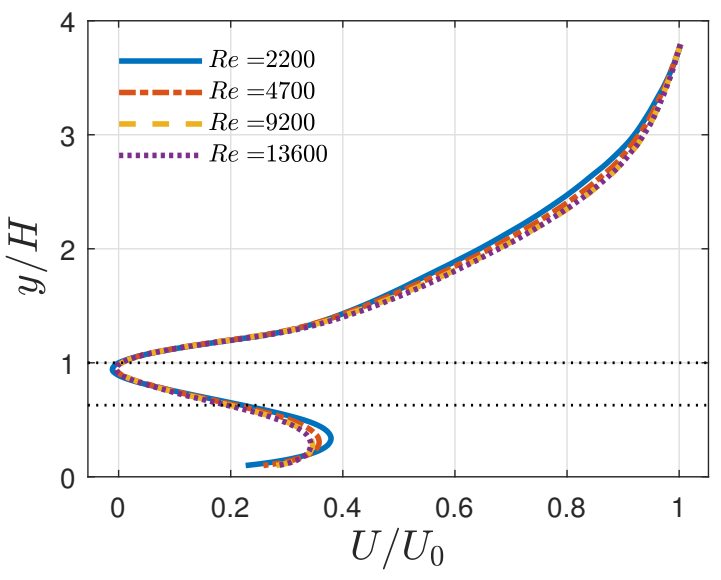

(a) $U / U_{0}$ : Streamwise mean velocity profile

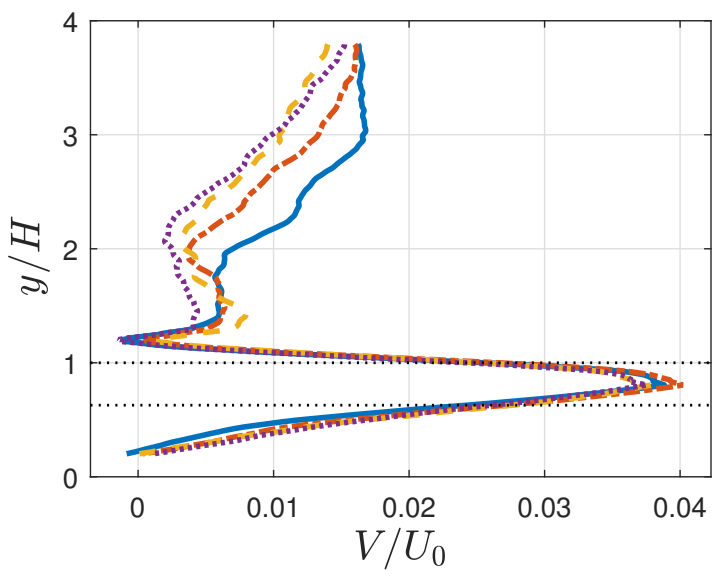

(b) $V / U_{0}$ : Vertical mean velocity profile

Figure 4.3: Reynolds number effect on normalized mean velocity profiles 
in $V / U_{0}$ affects how quickly the boundary layer develops over the farm. At lower speeds, Reynolds number has a larger apparent influence on the vertical flow in the upper region, due to either buoyancy or the panels themselves putting an upwards force on the flow.

The flow advecting in between the panels (i.e. in the direct wake) plays a significant role in the heat transfer of the panels. In this direct wake, the streamwise velocity has its maximum at the bottom of the panels, and the vertical velocity increases as $y / H$ increases. Throughout the direct wake, the vertical velocity has a positive value, demonstrating upwards movement. This suggests that the flow advecting in between the panels in fully developed flow is being pulled from underneath the panels as the sub-panel flow comes into contact with the inclined surface. This indicates that sub-panel flow, caused by the inclination of the solar panels thus plays an important role in the convective heat transfer and must be considered in the models.

Turbulence statistics measured at the four Reynolds numbers are shown in Fig. 4.4. The normal Reynolds stress distributions in the streamwise and vertical directions are shown in Figs. 4.4a and 4.4b. Variance maxima are observed in the region directly above the panels $1<y / H<2.2$ as expected for an obstructed shear flow or rough-wall boundary layer. Streamwise variances $\overline{u^{\prime} u^{\prime}} / U_{0}^{2}$ are on order of two times the wall-normal variances $\overline{v^{\prime} v^{\prime}} / U_{0}^{2}$. This indicates that the turbulence kinetic energy is predominately governed by the streamwise velocity fluctuations. The variances are mostly Reynolds number independent below the panels, but as much as $10 \%$ variation exists between the different Reynolds numbers.

The Reynolds normal and shear stress profiles indicate the shape of the wake 
caused by the solar farm. The upper and lower edges of the wake respectively are marked by local maxima in streamwise turbulence intensity at $y / H=0.7$ and $y / H=1.2$. At the center of the wake a minimum is observed, demonstrating that the center of the wake is at $y / H=1$. Increased values of streamwise turbulence are observed at the upper wake edge near $y / H=1.2$ compared to the wake lower edge due to higher wind speeds above the panels.

The Reynolds shear stress profile is shown in Fig. 4.4c. Elevated magnitudes of $-\overline{u^{\prime} v^{\prime}} / U_{0}^{2}$ are observed directly above the panel. Positive shear stress is observed in the direct wake of the panels from $0.5<y / H<1$. Comparing the profiles at varying Reynolds numbers, the lowest speed $R e=2200$ shows elevated wall-normal Reynolds stress and Reynolds shear stress magnitudes in the $1.2<y / H<3$, however as Reynolds number increases the stresses are equivalent within the measurement uncertainty. All of the Reynolds stress statistics show elevated magnitudes from $1<y / H<2.2$. In this region, the flow remains heavily influenced by interactions with the panels. The roughness of the solar panels to the flow generates high shear in the velocity profile, resulting in high turbulence levels at heights more than twice the height of the panels themselves.

Mean velocity and Reynolds normal and shear stresses at four input power levels $\left(q_{f}^{\prime \prime}=0.24,0.42,0.71,0.99\right)$ are presented in Fig. 4.5 and Fig. 4.6. Fig. 4.5a shows the normalized streamwise velocity profile. For the nominal case $q_{f}^{\prime \prime}=0.43$ below the panels, a maximum velocity of 0.35 to 0.4 is reached at a height of $y / H=0.3$. A strong velocity deficit is shown in the direct wake region, reaching a minimum of -0.02 at $y / H=1$. The contour plots indicate that this is due to a reversed flow region directly behind the panels. At $y / H=1.3$, an inflection point is observed, 

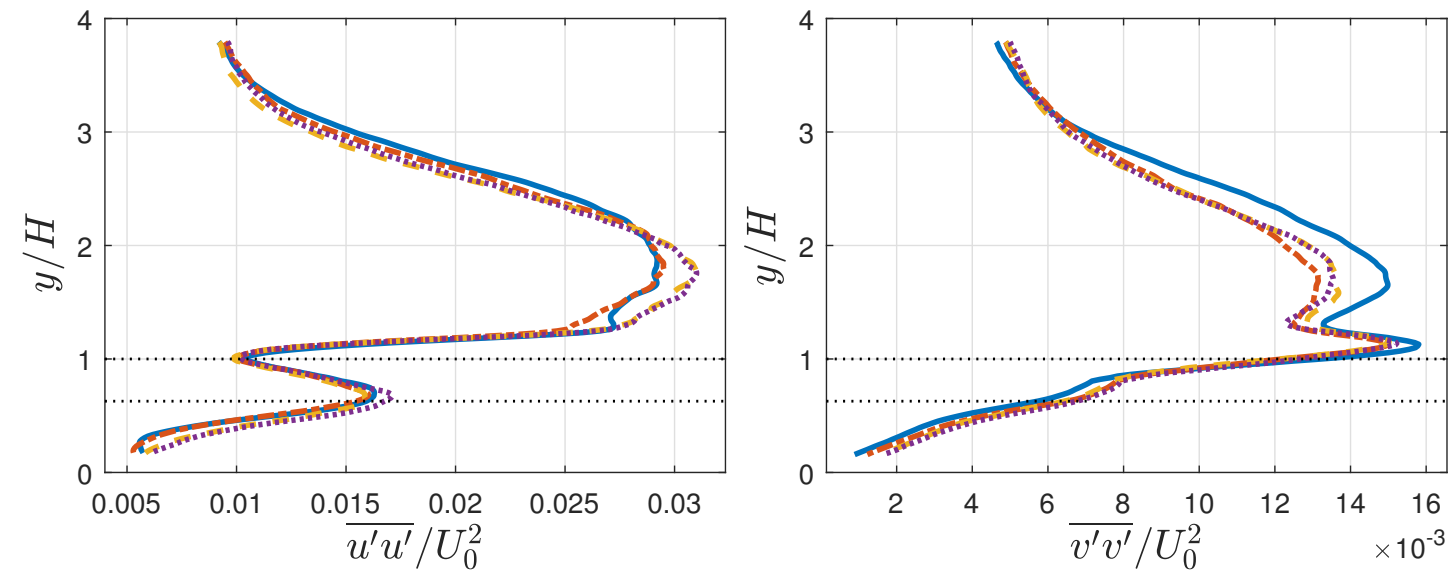

(a) $\overline{u^{\prime} u^{\prime}} / U_{0}^{2}$ : Streamwise Reynolds normal (b) $\overline{v^{\prime} v^{\prime}} / U_{0}^{2}$ : Vertical Reynolds normal stress stress

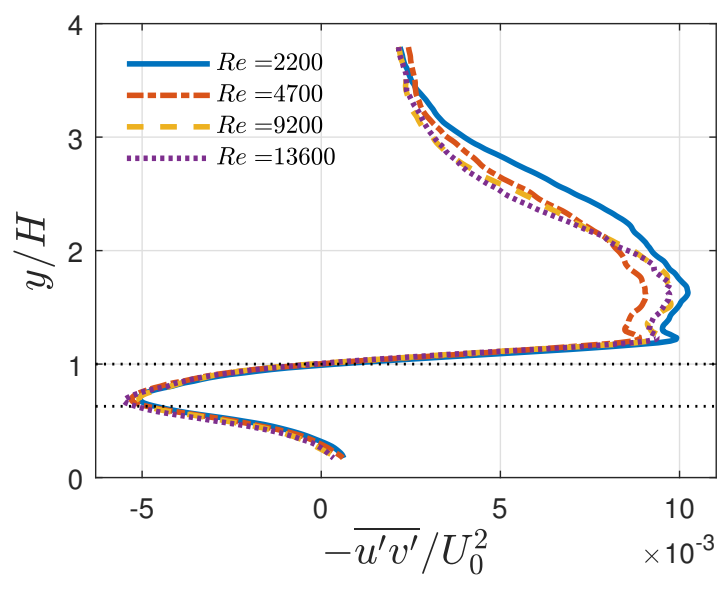

(c) $-\overline{u^{\prime} v^{\prime}} / U_{0}^{2}$ : Reynolds shear stress

Figure 4.4: Reynolds number effect on normalized Reynolds stresses

where $U / U_{0}$ transitions from highly sheared directly above the panels.

In the panel wake and directly above it $(0.6<y / H<1.3)$, the four heating values show nearly identical profiles, indicating that the streamwise flow is governed by the forced convection inflow and panel obstructions. However, the additional heat has an apparent effect on the flow in the sub-panel region $0<y / H<0.6$, where the $U / U_{0}$ increases with increasing power input, with the highest power $\left(q_{f}^{\prime \prime}\right.$ 


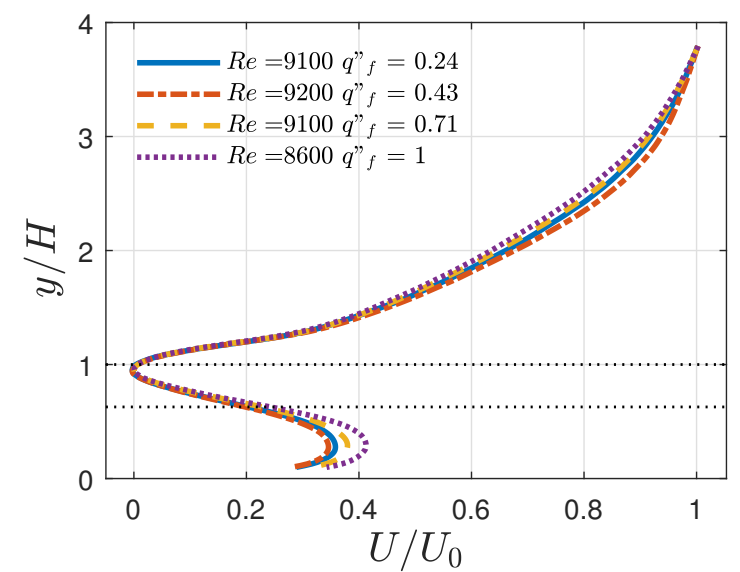

(a) $U / U_{0}$ : Streamwise mean velocity profile

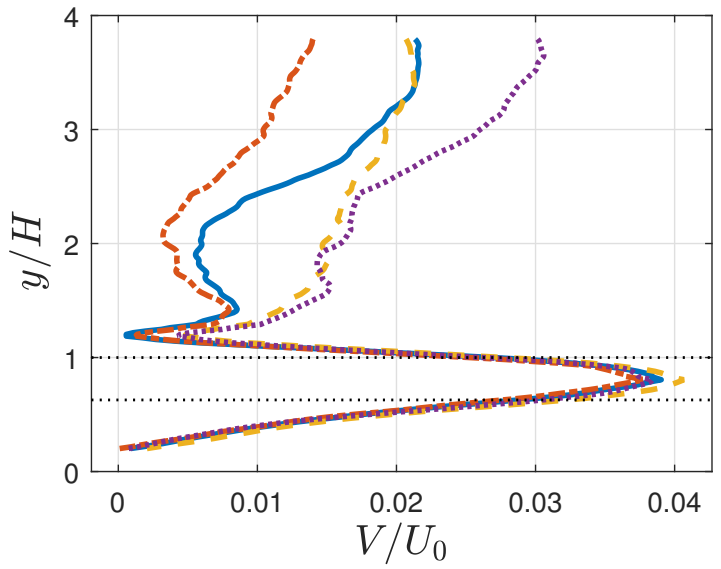

(b) $V / U_{0}$ : Vertical mean velocity profile

Figure 4.5: Input power level effect on normalized mean velocity profiles

$=1.00$ ) showing a $17 \%$ increase over the nominal case. The observed increase in speeds underneath the panels may be caused by unstable buoyancy interactions between the heated flow surrounding the panels and the cold tunnel floor. In the upper region $y / H>1.5$, increasing power shows lower $U / U_{0}$, however the difference in magnitude is not substantial. In this region, the decrease in $U / U_{0}$ from lowest and highest power levels was observed to be $4 \%$.

Profiles for normalized wall-normal velocity $V / U_{0}$ are shown in Fig. $4.5 \mathrm{~b}$ for four input power levels. Similar behavior is observed at all four power levels for $y / H<1.2$. Above $y / H>1.2$, close to where the velocity reaches an inflection point, substantial differences in $V / U_{0}$ are observed as a function of power level: a factor of 2.3 from lowest to highest power level. Increased heat released from the panels increases flow temperature, increasing the effect of buoyancy on the flow. Buoyancy is only strong enough to influence the flow outside of the direct wake of the panels, as the panels themselves are found to dictate the wall-normal velocity 

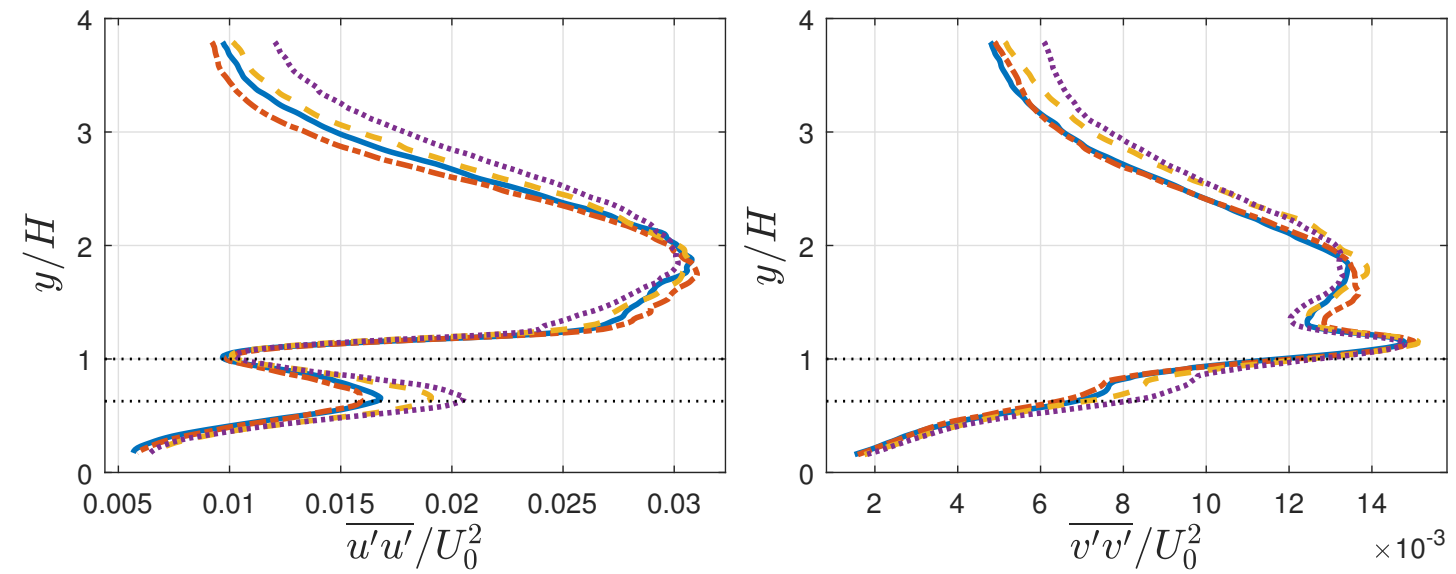

(a) $\overline{u^{\prime} u^{\prime}} / U_{0}^{2}$ : Streamwise Reynolds normal (b) $\overline{v^{\prime} v^{\prime}} / U_{0}^{2}$ : Vertical Reynolds Normal stress

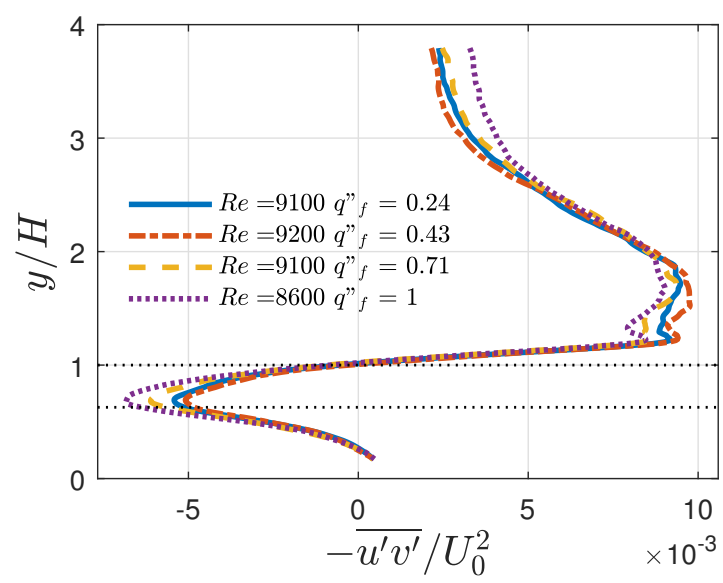

(c) $-\overline{u^{\prime} v^{\prime}} / U_{0}^{2}:$ In-plane Reynolds shear stress

Figure 4.6: Input power level effect on normalized Reynolds stresses

below $y / H<1.3$.

Figs. 4.6a, 4.6b, and 4.6c show the normalized normal and shear Reynolds stresses. At $y / H>2$, all three stresses increase with power level. Further, all three vary with power level in the direct wake region $0.5<y / H<1.0$, with the higher power showing the greatest magnitude stress. The increased sub panel velocity ob- 
served in Fig. 4.5a results in a higher velocity gradient in the wake region, resulting in higher levels of turbulence. Again, streamwise variances were on order of double the wall-normal variances. For streamwise variance, $\overline{u^{\prime} u^{\prime}} / U_{0}^{2}, q_{f}^{\prime \prime}=1$ showed an increase of $30 \%$ over the nominal case at $y / H=0.6$. This increase is directly caused by the increased sub-panel velocity shown in Fig. 4.5a.

Overall, these results show that over the range of Reynolds number tested, 2200 to 13500 , the convection heat transfer increases 2.9 times while the shape of the flow structure is largely Reynolds number independent above $R e>4700$.

\subsection{Turbulence Intensity Effects}

\subsubsection{Thermal}

Fig. 4.7 shows the effect of varying incoming turbulence intensity on $N u_{a d, u p}$ and $N u_{a d, l o}$ as generated by the single passive and two active grid settings with resulting average TI values of $11 \%, 18 \%$, and $16 \%$. The three settings are represented by different colors. Studies were performed at the nominal power input values, Reynolds number, and panel inclination angle. Each solid data point is the result of three separate experiments, shown by unfilled circles. Of the two settings governing the rotational speed of the wings on the active grid $\left(A G_{L o}\right.$ and $\left.A G_{H i}\right)$, the lower speed setting generated higher turbulence values (18\%), as slower rotational speeds enable larger integral length scales. The TI values shown in Fig. 4.7 are determined from the inflow profiles by averaging the TI between heights $2<y / H<4$.

On both upper and lower surfaces, average Nusselt number increases as turbulence intensity increases. For the upper surface, $N u_{a d, u p}$ ranges from 40.0 for the passive case, to 42.8 for the $A G_{L o}$. Lower surface $N u_{a d, l o}$ values were approximately 


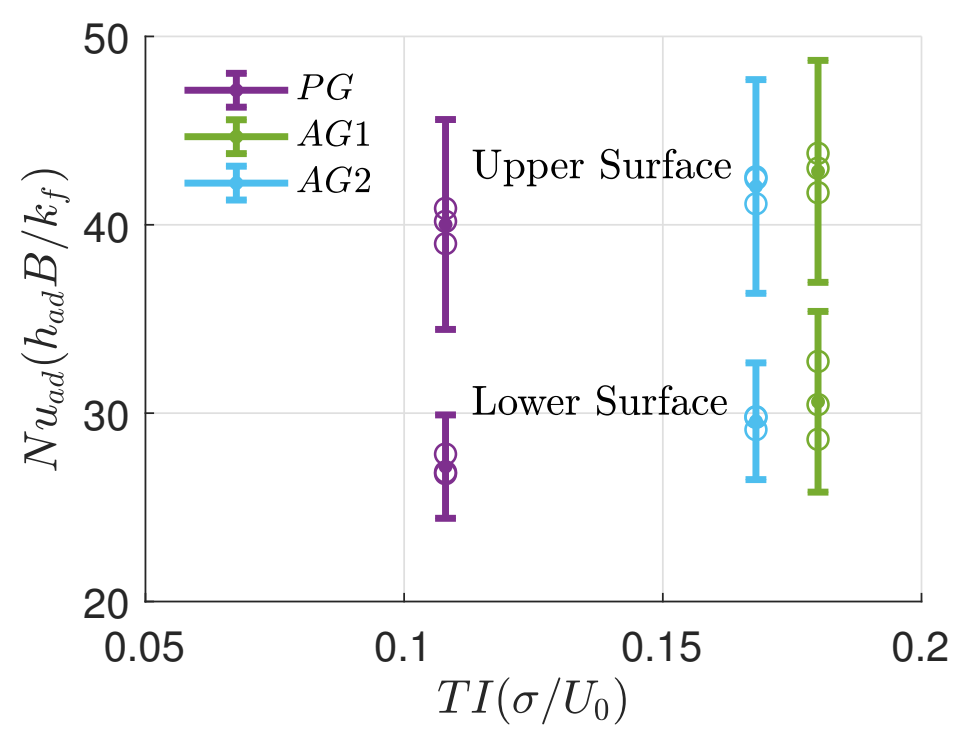

Figure 4.7: Turbulence effects on $N u_{a d, u p}$ and $N u_{a d, l o}$

$70 \%$ their upper surface counterparts. Both surfaces increase $7 \%$ from lowest turbulence values to highest. Measurement uncertainty averaged $13 \%$ on the upper surface and $12 \%$ on the lower.

The increases in $N u_{a d, u p}$ is well within the measurement uncertainty, and more cases would be required to define this increase more definitively. However, the apparent increase in $N u_{a d, u p}$ as TI increases is consistent with an increase in mixing between the flow at higher $y / H$ values and lower $y / H$ values. As turbulent fluctuations increase, more mixing occurs in the flow above the panels. This mixing increases interaction between the quickly advecting, lower temperature flow at the top of the scaled boundary layer and slowly advecting, higher temperature flow from near the surface of the panels, thus improving the heat transfer to the surrounding flow. However, this effect of TI on Nusselt number (7\% increase) is akin to an increase in Reynolds number from 2200 to 2600 , or $0.2 \mathrm{~ms}^{-1}$ at constant fluid properties and characteristic lengths. This suggests that enhancing bulk flow movement 
rather than increasing inflow turbulence may offer greater potential for temperature reduction.

\subsubsection{Flow field statistics}

The variation of the normalized streamwise velocity profile $U / U_{0}$ for different inflow turbulence values is shown in Fig. 4.8a. In sub-panel flow, all three settings reach their maximums at approximately $y / H=0.25$, and reach minimum values of 0.0 at $y / H=1.0$. The maximum variation between any of the settings at any wall-normal coordinate was $4 \%$; generally the three cases were in close agreement. The mean flow is governed by the panels rather than the inflow turbulence levels.

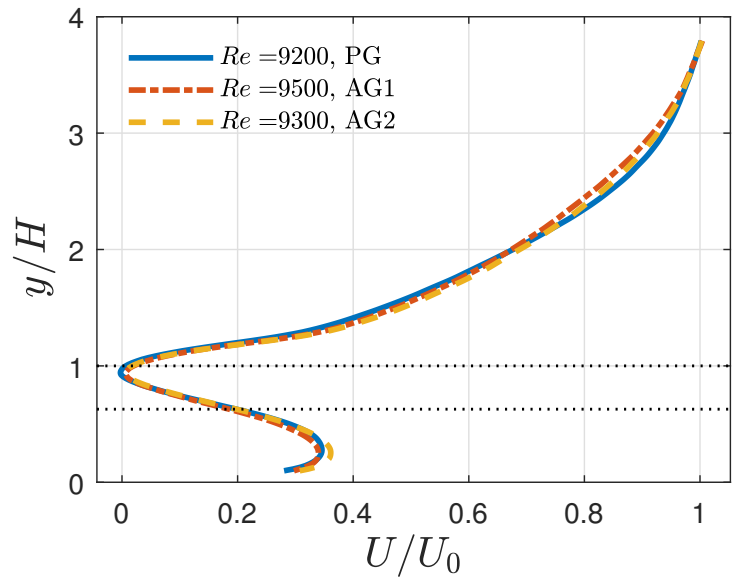

(a) Mean streamwise velocity

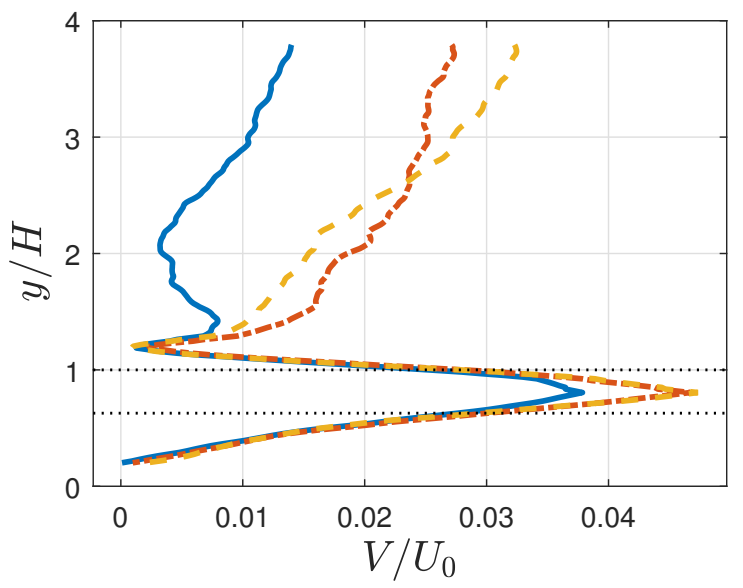

(b) Mean vertical velocity

Figure 4.8: Inflow turbulence effects on normalized mean velocity profiles

The variation of the normalized wall-normal velocity $V / U_{0}$ profile for different inflow turbulence values is shown in Fig. 4.8b, and the Reynolds stresses in Fig. 4.9. In contrast to the streamwise mean flow $U / U_{0}$, significant variations are shown between the passive grid and active grid cases. These differences are most notable 
in the direct-wake region $0.5<y / H<1$, and the upper-flow region $y / H>1.5$. In the direct-wake region of the panel $0.5<y / H<1$ shown in Fig. 4.8b, active grid $V / U_{0}$ increases $28 \%$ over the passive grid, and that difference increases to a $45 \%$ average above $y / H>1.5$.
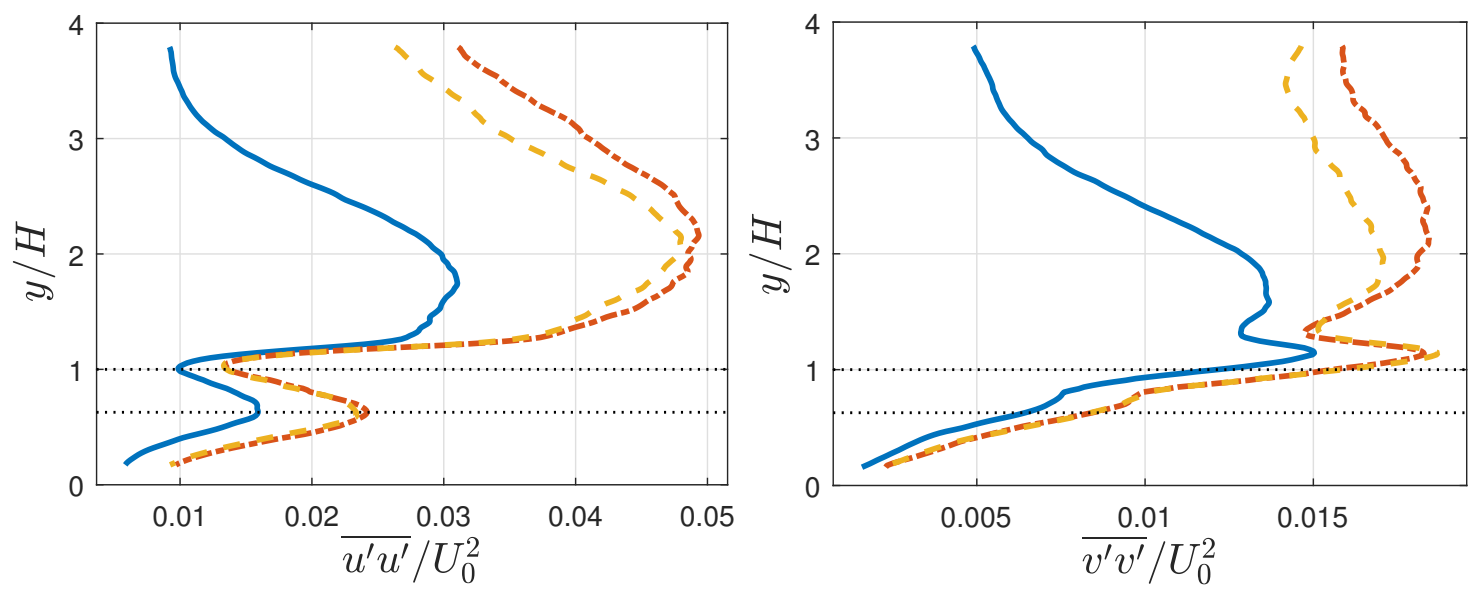

(a) $\overline{u^{\prime} u^{\prime}} / U_{0}^{2}$ : Streamwise Reynolds Normal (b) $\overline{v^{\prime} v^{\prime}} / U_{0}^{2}$ : Vertical Reynolds Normal stress

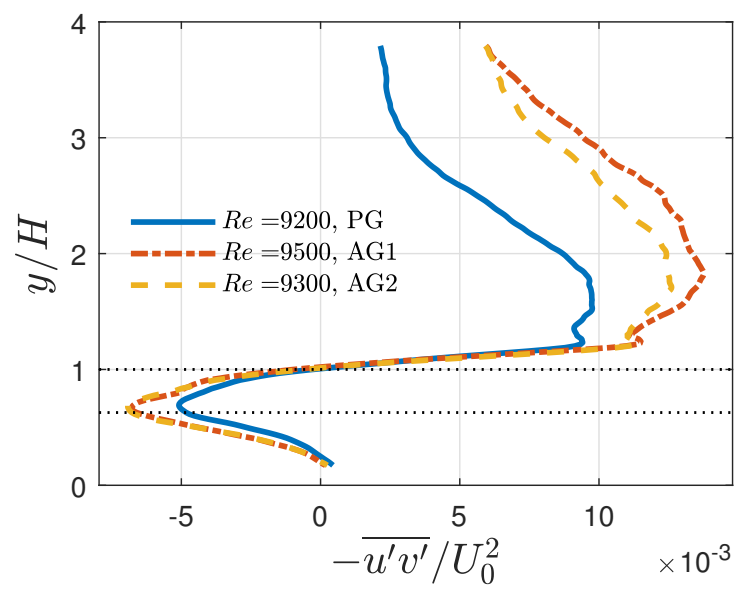

(c) $-\overline{u^{\prime} v^{\prime}} / U_{0}^{2}:$ In-plane Reynolds shear stress

Figure 4.9: Inflow turbulence effects on normalized Reynolds stresses

Figs. $4.9 \mathrm{a}$ and $4.9 \mathrm{~b}$ show the streamwise and wall-normal Reynolds stresses. 
Throughout the measured regime, the active grid cases show significantly higher Reynolds normal stresses than their passive grid counterpart. For the streamwise variance above $y / H>1.2$ the average increase is $125 \%$; for wall-normal variance it is $91 \%$. All quantities in Fig. 4.9 show a convergence between the different inflow cases just above the panel between $1<y / H<1.5$, suggesting that in this region the flow is governed by turbulence generated by the panels themselves, and less the inflow. However, below the panels at $y / H<0.6$, the effects of the inflow turbulence is still felt, shown by a $60 \%$ increase $\overline{u^{\prime} u^{\prime}} / U_{0}^{2}$ in Fig. $4.9 \mathrm{a}$ and indicating the effect of large scale turbulence.

For all four statistics shown in Fig. 4.9, above $y / H<1.2$ the $A G_{L o}$ and $A G_{H i}$ cases vary from the $P G$ case, but do not vary from each other. However, in Fig. 4.7 $N u_{a d, u p}$ continues to increase as TI increases. This suggests that another portion of the flow may be responsible for the increase in $N u_{a d, u p}$. It is hypothesized here that the Reynolds shear stress shown in Fig. 4.9c is responsible: above the panels $y / H>1.2,-\overline{u^{\prime} v^{\prime}} / U_{0}^{2}$ continues to increase as TI increases. Increasing Reynolds shear stress combined with bouyancy causes upward flow entrainment, thus mixing the flow more efficiently, and increasing the Nusselt number.

In summary, variations in the turbulent inflow are shown to offer increases of up to $7 \%$ to the convection heat transfer, and that the magnitude of possible changes is less than that demonstrated across the range of Reynolds numbers used in this study. 


\subsection{Panel Inclination Angle Effects}

Results of the variations in thermal characteristics, mean velocity, and Reynolds stresses for varying inclination angles are presented in section 4.3. For all inclination angles, the height of the bottom edge off of the floor does not change, meaning the panel pivots about the long edge lowest to the tunnel floor.

\subsubsection{Thermal}

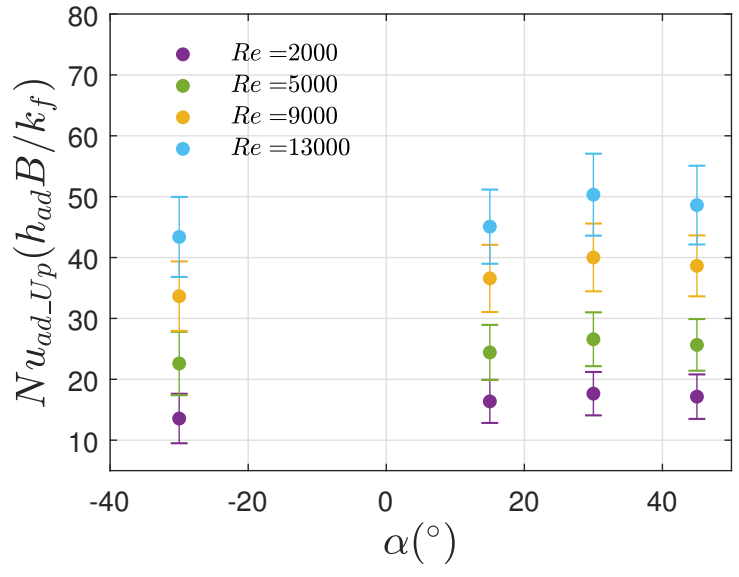

(a) Nusselt number on upper surface

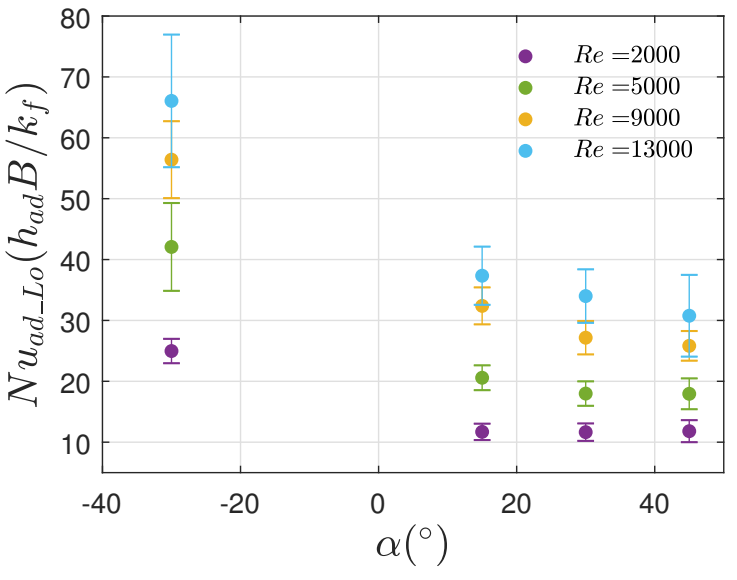

(b) Nusselt number on lower surface

Figure 4.10: Panel inclination angle effect on $N u_{a d, u p}$ and $N u_{a d, l o}$ at $q_{f}^{\prime \prime}=0.42$.

The variation of the panel inclination angle on convective heat transfer for the upper surface is shown in Fig. 4.10a. Four cases, one representing each Reynolds number, are shown for each angle. For the upper surface between $15^{\circ}$ and $30^{\circ}$, $N u_{a d, u p}$ rose for each Reynolds number. At the nominal case, $N u_{a d, u p}$ rose from 36.6 to 40.0 , representing a $9 \%$ increase. As Reynolds number increases, the change in $N u_{a d, u p}$ from $15^{\circ}$ to $30^{\circ}$ increased, rising from $7 \%, 8 \%, 9 \%$, and $10 \%$ at Reynolds 
numbers 2100, 4600, 9200, and 13500, respectively.

The same increase was not observed between $30^{\circ}$ and $45^{\circ}$. Fig. 4.10 a shows less than a $4 \%$ change between these angles, suggesting angle independence of $N u_{a d, u p}$ above approximately $30^{\circ}$. This is in agreement with Kaplani et al. who found $30^{\circ}$ the threshold for Nusselt number insensitivity [45]. Though the change observed is less than $4 \%$ and therefore is within the experimental uncertainty, a consistent $3-4 \%$ downwards trend is observed in each case.

It is suggested here that the inclination angle balances two aspects that affect the heat transfer. First, that increasing the angle increases the interactions with the overhead flow. $30^{\circ}$ inclination angles will interact more with the overhead flow than their $15^{\circ}$ counterparts, due to increased upwards propagation of the wake into the overhead flow. This increased interaction with lower temperature fluid will increase $N u_{a d, u p}$. However, past a certain angle, the effective blockage of the farm will increase (i.e. greater velocity deficit), impeding the flow more than it benefits from increased mixing. Further discussion continues with the analysis of mean velocity and Reynolds stresses below.

Fig. 4.10b shows $N u_{a d, l o}$ as a function of panel inclination at 4 different Reynolds numbers. Between $15^{\circ}$ and $30^{\circ}, N u_{a d, l o}$ exhibited opposite trends than $N u_{a d, u p}$. At each Reynolds number, the $15^{\circ}$ case showed higher $N u_{a d, l o}$ values than the $30^{\circ}$ counterpart. The nominal case showed a decrease in $N u_{a d, l o}$ from 32.4 to 27.2. Across the Reynolds number spectrum and starting from the lowest Reynolds value, from $15^{\circ}$ to $30^{\circ} N u_{a d, l o}$ decreased $4 \%, 14 \%, 19 \%$, and $10 \%$. Similar to $N u_{a d, u p}$, for the nominal case no discernible change was recorded for $N u_{a d, l o}$ between the $30^{\circ}$ and $45^{\circ}$ inclination angles. A decrease in $N u_{a d, l o}$ was observed when moving from $15^{\circ}$ to 
$30^{\circ}$ for Reynolds numbers greater than 5000. From a physical standpoint, as angle increases the rear surface is increasingly shadowed and operates in a larger velocity deficit region in the direct wake of the panels, with decreasing exposure to the sub-panel flow.

Panels angled at $-30^{\circ}$ effectively imitate the $+30^{\circ}$ case, but with the wind coming from the opposite direction of the solar farm. When compared with the $+30^{\circ}$ case, a decrease in $N u_{a d, u p}$ for the $-30^{\circ}$ case is seen in Fig. $4.10 \mathrm{a}$, ranging from $13 \%$ to $20 \%$ as Reynolds number increases. The absolute value change in $N u_{a d, u p}$ increases as Reynolds number decreases, but the percentage change remains similar within the bounds of the experimental uncertainty.

Comparing $N u_{a d, l o}$ for the $+30^{\circ}$ and $-30^{\circ}$ cases, a significant increase heat transfer was observed at $-30^{\circ}$. The nominal case rose from a $N u_{a d, l o}$ value of 27.2 to 56.4 , an increase of $108 \%$. Similar increases were observed for Reynolds numbers of 2100, 4600, and 13500, where $N u_{a d, l o}$ increases of $114 \%, 133 \%$, and $94 \%$ were recorded, respectively. When panels are angled negatively with respect to the direction of flow, flow is channeled underneath the panels, substantially increasing the subpanel velocity and overall convection efficiency. Further discussion continues with the analysis of mean velocity and Reynolds stresses below.

The ultimate goal of the present study is to identify promising avenues to reduce surface temperature in solar modules, and to better understand the effect of wind direction on the temperature, the surface temperatures taken at panel inclination angles of $+30^{\circ}$ and $-30^{\circ}$ were compared. Fig. 4.11 shows the difference between surface temperature and ambient temperature (measured at the pitot tube) throughout the solar array. Each graph contains four Reynolds numbers at $+30^{\circ}$ and $-30^{\circ}$. All 


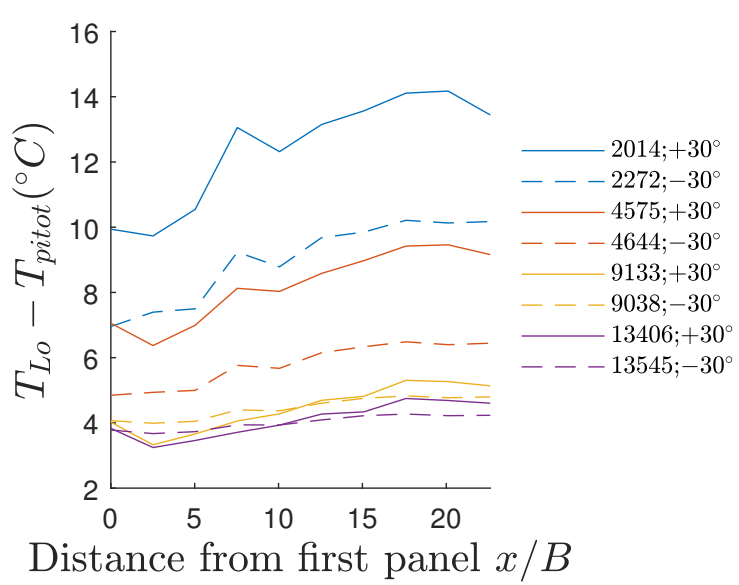

(a) Temperature rise on upper surface

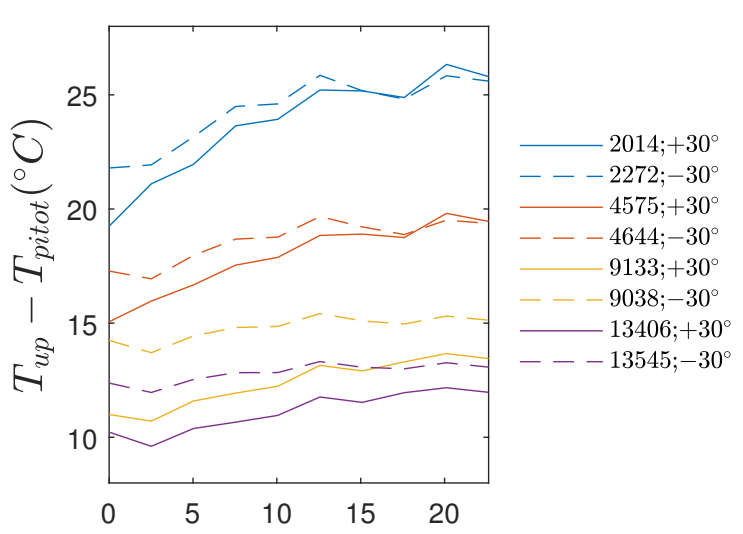

Distance from first panel $x / B$

(b) Temperature rise on lower surface

Figure 4.11: Surface Temperature Variations between $+30^{\circ}$ and $-30^{\circ}$ at $q_{f}^{\prime \prime}=0.42$

data shown is for $q_{f}^{\prime \prime}=0.42$ with a passive grid.

Fig. 4.11a shows the difference between the upper surface temperature and the ambient flow under fully steady state conditions when all panels are heated. For nominal conditions, from the first row to the last row, the $+30^{\circ}$ case surface temperature rises from $11.0^{\circ} \mathrm{C}$ to $13.5^{\circ} \mathrm{C}$. The $-30^{\circ}$ case is notably warmer, beginning at $14.2^{\circ} \mathrm{C}$ and rising to $15.1^{\circ} \mathrm{C}$. All Reynolds numbers exhibit similar trends, with the notable difference that as Reynolds number decreases the difference between the $+30^{\circ}$ case and the $-30^{\circ}$ case disappears. Further, as Reynolds number increases, the difference between the first and last panel increases for both the $+30^{\circ}$ and $30^{\circ}$ cases. For the lowest Reynolds number at $+30^{\circ}$ the $T_{\text {row } 10}-T_{\text {row } 1}=6.3^{\circ} \mathrm{C}$, and as Reynolds number increases the $\Delta T$ decreases to $4.4^{\circ} \mathrm{C}, 2.5^{\circ} \mathrm{C}$, and $1.8^{\circ} \mathrm{C}$. At higher Reynolds numbers, it is observed that the second panel is the coolest, an effect of the first panel generating a turbulent wake.

Lower surface temperatures show markedly different trends that correspond to 
the increase in $N u_{a d, l o}$ that was observed in Fig. 4.10b. The higher Reynolds numbers show smaller differences between $+30^{\circ}$ and $-30^{\circ}$ when compared to the lower Reynolds numbers. The largest difference observed at $R e=9000$, and $R e=$ 13500 is $0.7^{\circ} \mathrm{C}$, and the mean difference is negligible. However, lower Reynolds numbers show substantial differences throughout all ten rows. At $R e=4600$, the $-30^{\circ}$ case is an average of $2.4^{\circ} \mathrm{C}$ lower, and for $R e=2300$, the $-30^{\circ}$ case is an average of $3.4^{\circ} \mathrm{C}$ lower.

It is quite notable that the variance between the cases dissipates by row 7 in Fig. 4.11a, but the gains shown in the lower surface in Fig. 4.11b are perpetuated throughout the farm. It suggests that the mechanism responsible for the lower surface cooling does not inherently negatively effect the upper surface, and vice versa. Further discussion on potential mechanisms is found in the discussion on mean velocity and Reynolds stresses.

\subsubsection{Flow field statistics}

The normalized streamwise velocity $U / U_{0}$ is shown in Fig $4.12 \mathrm{a}$, and is compared across the different panel inclination angles. Each figure contains the black horizontal dotted lines designating the top and bottom of the physical location of the panels angled at $30^{\circ}$. At the wall-normal coordinate $y / H=0.35$, maximum $U / U_{0}$ increases from 0.29 , to $0.37,0.41$, and 0.47 as $\alpha$ changes from $15^{\circ}$ to $45^{\circ}, 30^{\circ}$, and $-30^{\circ}$. The $-30^{\circ}$ case maintains higher speeds very close to the bottom edge of the panels while the $15^{\circ}, 30^{\circ}$, and $45^{\circ}$ cases show a larger decrease in speed adjacent to the underside of the panels. At $y / H=0.6$, the $-30^{\circ}$ case $U / U_{0}$ increases over the other three angles $40 \%$ to $82 \%$. 
Increasing inclination angle increases the velocity deficit in the wake of the panels, due to an increase in projected area and increased pressure drag. The deficit measured relative to the freestream flow increase from $\left(U_{\infty}-U_{\text {min }}\right) / U_{\infty}=92.5 \%$ at $15^{\circ}, 102 \%$ at $30^{\circ}$, and $104 \%$ at $45^{\circ}$. As angles increase the drag approaches that of a flat plat in perpendicular flow. In the post stall region, the drag on a flat plate follows sinusoidal curve due to the pressure drag dominating skin friction drag at higher angles [46]. Increases at higher angles are only caused by small increased in area projected normal to the flow. The deficit shown in Fig. 4.12a follows this trend, where the deficit is larger between $15^{\circ}$ and $30^{\circ}$ than between $30^{\circ}$ and $45^{\circ}$. For all angles, this increased drag influences the flow up to $y / H=3$.

The vertical velocity component is shown in Fig. 4.12b. Inclination angles $15^{\circ}$, $30^{\circ}$, and $45^{\circ}$ behave similarly across the wall-normal coordinate. Starting from near $-1 \%$ of the freestream $U_{0}$, they increase rapidly to $2.5 \%, 4 \%$, and $5.8 \%$ at $y / H=0.8$ for the $15^{\circ}, 30^{\circ}$, and $45^{\circ}$ cases respectively. Below the panels the $15^{\circ}$ case shows

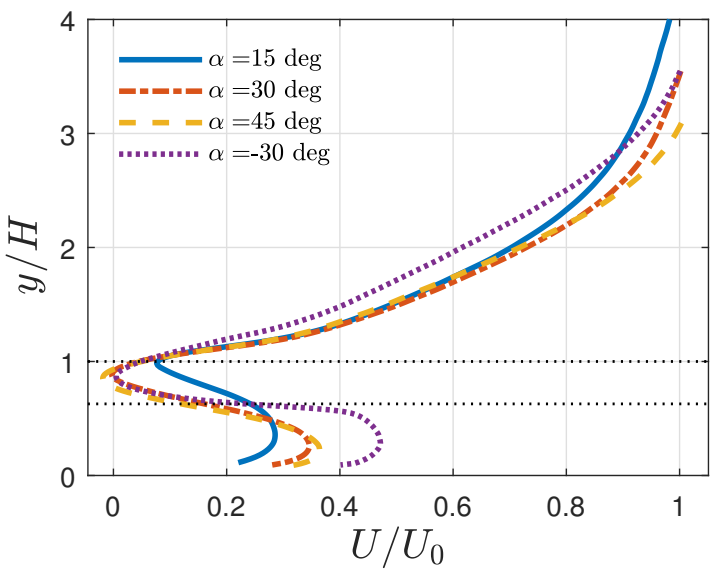

(a) $\frac{U}{U_{0}}$ : Streamwise mean velocity profile

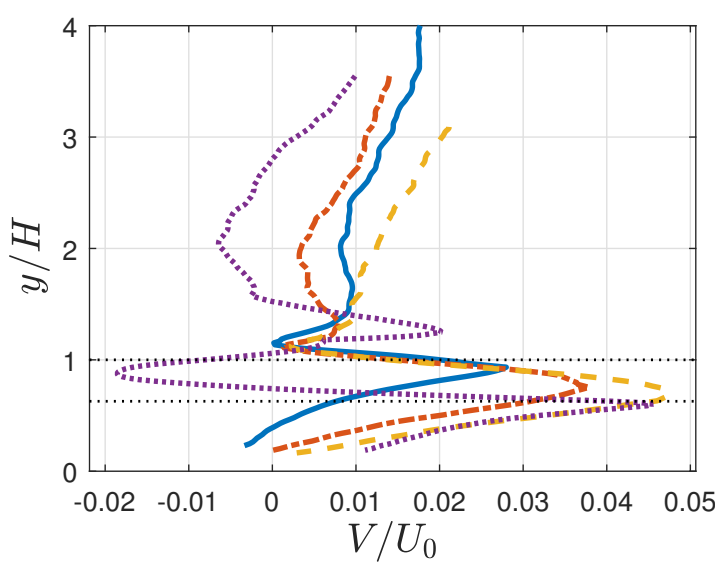

(b) $\frac{V}{U_{0}}$ : Wall-normal mean velocity profile

Figure 4.12: Panel inclination angle effect on normalized mean velocity profiles 
higher $V / U_{0}$, but in the wake of the panels this reverses, and by $y / H=1$, the $45^{\circ}$ case shows the larger $V / U_{0}$, which is related to the large streamwise velocity deficit in the wake. In the upper flow region, the velocity ranges from $-1 \%$ to $-3 \%$ for the three positive inclination angle cases. By contrast, $V / U_{0}$ for the $-30^{\circ}$ case has a much sharper increase and decrease in the $y / H<1$ range, dropping to $-2.6 \%$ at $y / H=1$. The maximum $V / U_{0}$ for the $-30^{\circ}$ case reaches $-2.6 \%$, compared to $-1.3 \%$ for the other three cases.

The extent of the cooling that can be accomplished with any given array configuration depends on the way in which incoming flow is divided into array flow and bypass flow. Array flow is that passing flow which traverses underneath and between the panels while bypass flow is that incoming flow which does not or only slightly interacts with the array. When angled at $-30^{\circ}$, the panels effectively act as downward baffles, taking flow that would have been deflected upwards and channeling it underneath the panels. This is demonstrated in Fig. 4.12b, where the normalized wall-normal velocity $V / U_{0}$ shows a steep negative gradient throughout the wake of the panel, such that at $y / H=1, V / U_{0}$ is almost $-100 \%$ of $V / U_{0}$ in the $+30^{\circ}$ case. This is an indication that the $+30^{\circ}$ case deflects the flow upwards, while the $-30^{\circ}$ pulls it down. When comparing the $U / U_{0}$ above the panels, a decrease is observed in the upper flow region, but the $+30^{\circ} U / U_{0}$ does not surpass the $-30^{\circ}$ case until $y / H=1.2$. The net effect is that an increased volume of fluid interacts with the panels compared with the $+30^{\circ}$ case (i.e. an increase in array flow relative to bypass flow).

Reynolds normal and shear stresses are shown in Figs. 4.13a, 4.13b, and 4.13c. Both Reynolds normal stresses exhibit consistent trends from $15^{\circ}$ to $45^{\circ}$, generally 
increasing by similar magnitudes as inclination angle increments. A typical case is observed in Fig. 4.13a at $y / H=0.6$. As panel inclination angle increases from $15^{\circ}$ to $30^{\circ}$ and $15^{\circ}$ to $45^{\circ}$, the streamwise variance increases $60 \%$ and $162 \%$ respectively. Increased drag due to increased inclination angle generates more turbulent perturbations, which dissipate the mean kinetic energy of the flow. As observed in other cases, the streamwise variance is on order of twice as large as the wall-normal variance.

Improved heat transfer is considered to be a balance between increasing the interaction with passing fluid through increased turbulent mixing, and decreasing the velocity near and through the arrays leading to less efficient convection. At $45^{\circ}$, the flow is impeded substantially, decreasing the benefit to heat transfer. However, at $15^{\circ}$ lower $N u_{a d, u p}$ is observed, likely because the turbulent interaction with the flow is reduced. This lack of interaction is indicated by much lower Reynolds stress values in the region $y / H=1$ to 2 . In this case, the passing flow continues to move quickly, but it does not interact with or increase panel convection.

The $-30^{\circ}$ case exhibits significantly higher stresses across the spectrum, often greater than a $100 \%$ increase over the second highest stress. At $y / H=1.5$, the streamwise variance in Fig. 4.13a shows a 92\% increase and the Reynolds shear stress in Fig. 4.13c a 145\% increase over any other cases. This leads to increased upward entrainment near the top of the panels and increased thermal mixing. A large negative shear stress near $y / H=0.7$ in the direct wake of the panels demonstrates the angle of the panels directs the flow below the panels and increasing the sub-panel flow observed in Fig. 4.12a.

Overall, these results show that increasing panel inclination angle acts to improve 
convection up to an angle of approximately $30^{\circ}$, and that substantial increases in heat transfer are observed with a $180^{\circ}$ change in wind direction.
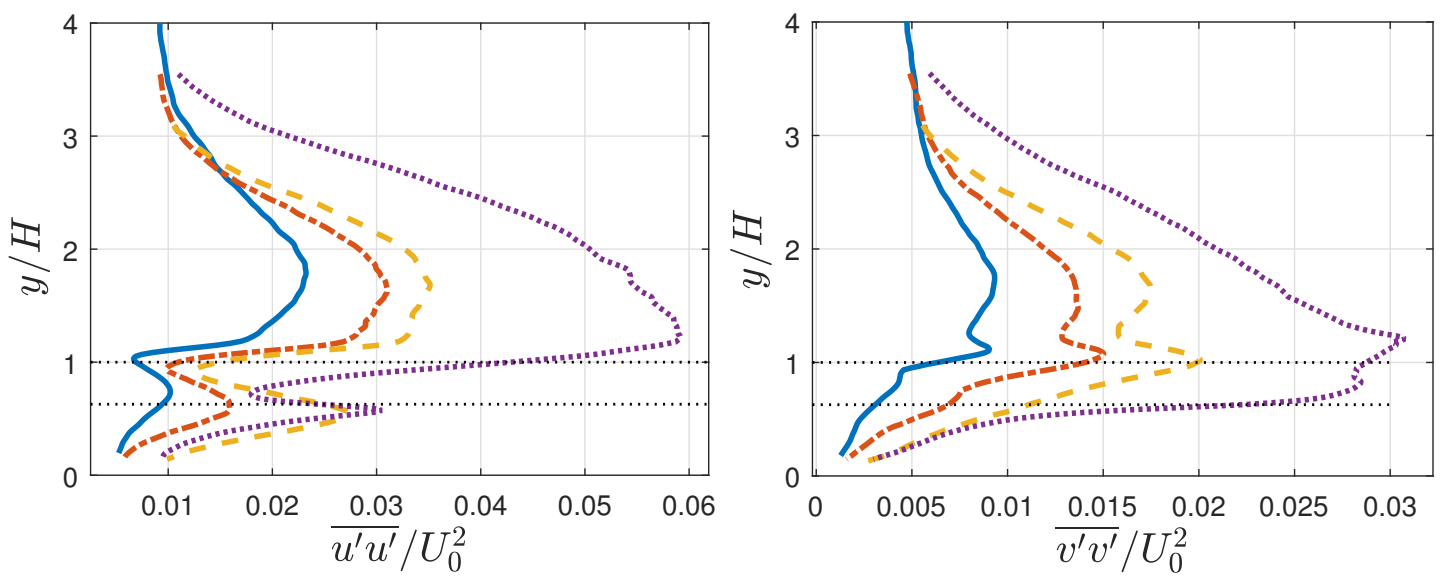

(a) $\overline{u^{\prime} u^{\prime}} / U_{0}^{2}$ : Streamwise Reynolds normal (b) $\overline{v^{\prime} v^{\prime}} / U_{0}^{2}$ : Vertical Reynolds normal stress

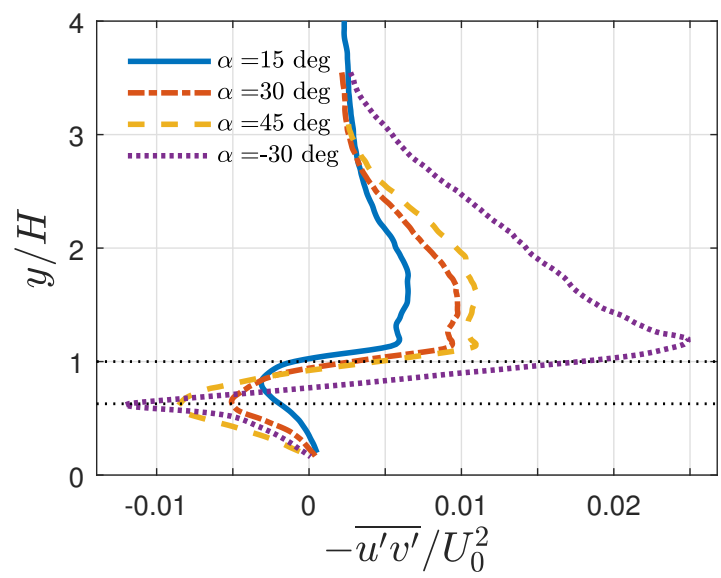

(c) $-\overline{u^{\prime} v^{\prime}} / U_{0}^{2}:$ In-plane Reynolds shear stress

Figure 4.13: Panel inclination angle effect on normalized Reynolds stresses 


\section{Chapter 5}

\section{Conclusions}

The research reported here represents the first controlled investigation of heat transfer and fluid flow in infinite PV solar arrays. An experiment was designed to explore the effect of fluid flow over and through a large PV solar array on the heat transfer properties of that array. To simulate large outdoor solar arrays in a controlled environment, forty model solar panels, represented as heated surfaces, were organized into a ten row array and tested in a wind tunnel. Reynolds number, inflow turbulence intensity, and panel inclination angle were varied to determine their effect on the heat transfer coefficient.

It was demonstrated that the convective heat transfer coefficient increased with Reynolds number for both the upper and lower surfaces. Nusselt number increased by a factor of 2.9 across the range of Reynolds numbers tested (2200 to 13500). Increased inflow turbulence intensity increased the Nusselt number on both the front and rear surfaces by as much as $7 \%$, however these effects were small compared to the changes observed with increases in Reynolds number. Increasing panel inclination angle caused an increase in $N u_{a d, u p}$ on the upper surface from $15^{\circ}$ to $30^{\circ}$, but leveled out from $30^{\circ}$ to $45^{\circ}$. The opposite trend was observed in the lower surface, decreasing $N u_{a d, l o}$ from $15^{\circ}$ to $30^{\circ}$ and again leveling out from $30^{\circ}$ to $45^{\circ}$. 
These results point towards a tradeoff between flow obstructions increasing the velocity deficit and the increased mixing that accompanies increased upward wake propagation as panel inclination angle increases.

The $-30^{\circ}$ case showed the largest effect on heat transfer on the lower surface. Compared to the $+30^{\circ}$ case for row 8 , the upper surface heat transfer coefficient decreased by approximately $25 \%$, while the lower surface heat transfer coefficient increased by $108 \%$. These substantial changes indicate that altering the flow field with deflectors, and designing a solar farm to enhance sub-panel flow speed can offer substantial reductions in solar farm surface temperature and LCOE.

The study of heat transfer in infinite solar arrays is important to improve models and develop new strategies for solar panel temperature reduction. Improved models used for research will allow scientists to identify high impact areas of future research, and improved industry models will allow for better cost estimates and reduced uncertainty in LCOE for a proposed project. New cooling strategies could offer low-cost, retrofittable solutions to existing and new farms, reducing the cost of energy itself through longer lasting panels and improved efficiency. This becomes increasingly relevant as adoption of solar technology spreads and materials bump up against their efficiency limits. Lowering the LCOE of solar energy is a key step towards wider adoption of the technology, and an essential part of mitigating the worst effects of climate change. 


\section{Chapter 6}

\section{Future Work}

This experiment lays the groundwork for future work exploring enhancements to convection cooling of large arrays of PV panels. Near term work will be aimed at changing the spacing between the panels, the distance the panels are from the ground, varying the height, changing the angle of incidence of the rows, various non symmetrical layouts, and adding flow deflectors. Flow deflectors may be placed above the farm, channeling flow downwards, or even within the farm, as was shown to be effective in the $-30^{\circ}$ case. Natural convection enhancements will be explored more completely to understand potential benefits of array optimization for the many low wind speed sites where these arrays are located. Comparisons with both numerical simulations and real world farms is currently underway. Economic analysis will accompany future designs. The goal of all future experiments will be geared towards reducing the surface temperature of the farms with practical cost-effective solutions, and doing a small part to mitigate climate change. 


\section{Bibliography}

[1] E Skoplaki and JA Palyvos. On the temperature dependence of photovoltaic module electrical performance: A review of efficiency/power correlations. Solar Energy, 83(5):614-624, 2009.

[2] M Hasanuzzaman, ABMA Malek, MM Islam, AK Pandey, and NA Rahim. Global advancement of cooling technologies for pv systems: A review. Solar Energy, 137:25-45, 2016.

[3] MA Green and SP Bremner. Energy conversion approaches and materials for high-efficiency photovoltaics. Nature Materials, 16(1):23, 2017.

[4] S Kurtz, K Whitfield, G TamizhMani, M Koehl, D Miller, J Joyce, J Wohlgemuth, N Bosco, M Kempe, and T Zgonena. Evaluation of high-temperature exposure of photovoltaic modules. Progress in Photovoltaics: Research and Applications, 19(8):954-965, 2011.

[5] DH Otth and RG Ross. Assessing photovoltaic module degradation and lifetime from long term environmental tests. In 29th Institute of Environmental Sciences Technical Meeting, Los Angeles, CA, pages 121-126, 1983.

[6] X Wang, L Kurdgelashvili, J Byrne, and A Barnett. The value of module efficiency in lowering the levelized cost of energy of photovoltaic systems. Renewable and Sustainable Energy Reviews, 15(9):4248-4254, 2011.

[7] R Vaillon, O Dupré, RB Cal, and M Calaf. Pathways for mitigating thermal losses in solar photovoltaics. Scientific Reports, 8(1):13163, 2018.

[8] TJ Silverman, MG Deceglie, I Subedi, NJ Podraza, IM Slauch, VE Ferry, and I Repins. Reducing operating temperature in photovoltaic modules. IEEE Journal of Photovoltaics, 2018.

[9] L Zhu, A Raman, K Wang, M Anoma, and S Fan. Radiative cooling of solar cells. Optica, 1(1):32-38, 2014. 
[10] T Chow. A review on photovoltaic/thermal hybrid solar technology. Applied Energy, 87(2):365-379, 2010.

[11] HG Teo, PS Lee, and M Hawlader. An active cooling system for photovoltaic modules. Applied Energy, 90(1):309-315, 2012.

[12] DJ Yang, ZF Yuan, PH Lee, and HM Yin. Simulation and experimental validation of heat transfer in a novel hybrid solar panel. International Journal of Heat and Mass Transfer, 55(4):1076-1082, 2012.

[13] EM Sparrow, JE Niethammer, and A Chaboki. Heat transfer and pressure drop characteristics of arrays of rectangular modules encountered in electronic equipment. International Journal of Heat and Mass Transfer, 25(7):961-973, 1982 .

[14] W De Soto, SA Klein, and WA Beckman. Improvement and validation of a model for photovoltaic array performance. Solar Energy, 80(1):78-88, 2006.

[15] DL King, JA Kratochvil, and WE Boyson. Photovoltaic array performance model. United States. Department of Energy, 2004.

[16] MM Rahman, M Hasanuzzaman, and NA Rahim. Effects of various parameters on pv-module power and efficiency. Energy Conversion and Management, 103:348-358, 2015.

[17] S Mekhilef, R Saidur, and M Kamalisarvestani. Effect of dust, humidity and air velocity on efficiency of photovoltaic cells. Renewable and Sustainable Energy Reviews, 16(5):2920-2925, 2012.

[18] S Armstrong and WG Hurley. A thermal model for photovoltaic panels under varying atmospheric conditions. Applied Thermal Engineering, 30(11):1488$1495,2010$.

[19] EPJA Skoplaki and JA Palyvos. Operating temperature of photovoltaic modules: A survey of pertinent correlations. Renewable Energy, 34(1):23-29, 2009.

[20] EM Sparrow. Effect of finite width on heat transfer and fluid flow about an inclined rectangular plate. Journal of Heat Transfer, 101:199, 1979.

[21] S Kumar and SC Mullick. Wind heat transfer coefficient in solar collectors in outdoor conditions. Solar Energy, 84(6):956-963, 2010.

[22] E Sartori. Convection coefficient equations for forced air flow over flat surfaces. Solar Energy, 80(9):1063-1071, 2006. 
[23] EM Sparrow, JS Nelson, and WQ Tao. Effect of leeward orientation, adiabatic framing surfaces, and eaves on solar-collector-related heat transfer coefficients. Solar Energy, 29(1):33-41, 1982.

[24] S Sharples and PS Charlesworth. Full-scale measurements of wind-induced convective heat transfer from a roof-mounted flat plate solar collector. Solar Energy, 62(2):69-77, 1998.

[25] JLA Francey and J Paraioannou. Wind-related heat losses of a flat-plate collector. Solar Energy, 35(1):15-19, 1985.

[26] PI Cooper. The effect of inclination on the heat loss from flat-plate solar collectors. Solar Energy, 27(5):413-420, 1981.

[27] A Hobbi and K Siddiqui. Experimental study on the effect of heat transfer enhancement devices in flat-plate solar collectors. International Journal of Heat and Mass Transfer, 52(19):4650-4658, 2009.

[28] F Kreith and JF Kreider. Principles of solar engineering. Washington, DC, Hemisphere Publishing Corp., 1978. 790 p., 1978.

[29] SV Garimella and PA Eibeck. Heat transfer characteristics of an array of protruding elements in single phase forced convection. International Journal of Heat and Mass Transfer, 33(12):2659-2669, 1990.

[30] H Wu and S Perng. Effect of an oblique plate on the heat transfer enhancement of mixed convection over heated blocks in a horizontal channel. International Journal of Heat and Mass Transfer, 42(7):1217-1235, 1999.

[31] S Yang. A numerical investigation of heat transfer enhancement for electronic devices using an oscillating vortex generator. Numerical Heat Transfer: Part A: Applications, 42(3):269-284, 2002.

[32] H Ay, J Jang, and J Yeh. Local heat transfer measurements of plate finned-tube heat exchangers by infrared thermography. International Journal of Heat and Mass Transfer, 45(20):4069-4078, 2002.

[33] FL Test, RC Lessmann, and A Johary. Heat transfer during wind flow over rectangular bodies in the natural environment. Journal of Heat Transfer, 103(2):262-267, 1981. 
[34] MK Chyu, YC Hsing, and V Natarajan. Convective heat transfer of cubic fin arrays in a narrow channel. TRANSACTIONS-AMERICAN SOCIETY OF MECHANICAL ENGINEERS JOURNAL OF TURBOMACHINERY, 120:362367, 1998.

[35] AM Anderson and RJ Moffat. Direct air cooling of electronic components: reducing component temperatures by controlled thermal mixing. J. Heat Transfer, 113(5), 1991.

[36] S Garimella and P Eibeck. Fluid dynamic characteristics of the flow over an array of large roughness elements. In Thermal Phenomena in Electronic Systems, 1992. I-THERM III, InterSociety Conference on, pages 102-109. IEEE, 1992.

[37] T Young and K Vafai. Convective flow and heat transfer in a channel containing multiple heated obstacles. International Journal of Heat and Mass Transfer, 41(21):3279-3298, 1998.

[38] R Moffat and A Ortega. Direct air-cooling of electronic components. Advances in Thermal Modeling of Electronic Components and Systems, 1:129-282, 1988.

[39] X Wang and L Castillo. Asymptotic solutions in forced convection turbulent boundary layers. Journal of Turbulence, 4:6-6, 2003.

[40] G Freedland. Investigation of jet dynamics in cross-flow: Quantifying volcanic plume behavior. 2016.

[41] RB Cal, J Lebrón, L Castillo, HS Kang, and C Meneveau. Experimental study of the horizontally averaged flow structure in a model wind-turbine array boundary layer. Journal of Renewable and Sustainable Energy, 2(1):013106, 2010.

[42] RS Figliola and D Beasley. Theory and design for mechanical measurements. John Wiley \& Sons, 2015.

[43] SA Kalogirou. Solar energy engineering: processes and systems. Academic Press, 2013.

[44] FP Incropera, AS Lavine, TL Bergman, and DP DeWitt. Fundamentals of heat and mass transfer. Wiley, 2007.

[45] E Kaplani and S Kaplanis. Thermal modelling and experimental assessment of the dependence of pv module temperature on wind velocity and direction, module orientation and inclination. Solar Energy, 107:443-460, 2014. 
[46] LA Viterna and RD Corrigan. Fixed pitch rotor performance of large horizontal axis wind turbines. 1982. 
Appendices 


\section{A Data Reduction Equations}

$$
\begin{aligned}
& q_{u p}^{\prime \prime}=q_{c o n v, u p}^{\prime \prime}+q_{r a d, u p}^{\prime \prime} \\
& =q_{\text {conv }, u p}^{\prime \prime}+q_{\text {rad,up,ceil }}^{\prime \prime}+q_{\text {rad,up,pan }}^{\prime \prime}+q_{\text {rad,up }, \text { flr }}^{\prime \prime} \\
& =h_{\text {ad,up }}\left(T_{u p}-T_{a d, u p}\right)+F_{\text {up_ceil }} \cdot \sigma \epsilon_{u p}\left(T_{u p}^{4}-T_{\text {ceil }}^{4}\right) \\
& +F_{\text {up_pan }} \cdot \sigma \epsilon_{\text {up }}\left(T_{u p}^{4}-T_{\text {lo }}^{4}\right)+F_{\text {up_flr }} \cdot \sigma \epsilon_{\text {up }}\left(T_{\text {up }}^{4}-T_{\text {floor }}^{4}\right) \text {. } \\
& q_{l o}^{\prime \prime}=q_{\text {conv }, l o}^{\prime \prime}+q_{\text {rad,lo }}^{\prime \prime} \\
& =q_{\text {conv }, l o}^{\prime \prime}+q_{\text {rad,lo,ceil }}^{\prime \prime}+q_{\text {rad,lo,pan }}^{\prime \prime}+q_{\text {rad,lo,flr }}^{\prime \prime} \\
& =h_{a d, l o}\left(T_{l o}-T_{a d, l o}\right)+F_{l o \_c e i l} \cdot \sigma \epsilon_{l o}\left(T_{l o}^{4}-T_{c e i l}^{4}\right) \\
& +F_{l o \_p a n} \cdot \sigma \epsilon_{l o}\left(T_{l o}^{4}-T_{u p}^{4}\right)+F_{l o_{-} f l r} \cdot \sigma \epsilon_{l o}\left(T_{l o}^{4}-T_{\text {floor }}^{4}\right) \text {. } \\
& {\left[\begin{array}{c}
q_{e} \\
\frac{T_{u p}}{R_{f n t}} \\
\frac{T_{l o}}{R_{b c k}}
\end{array}\right]=\left[\begin{array}{ccc}
1 & 1 & 0 \\
-1 & 0 & \frac{1}{R_{f n t}} \\
0 & -1 & \frac{1}{R_{b c k}}
\end{array}\right] \times\left[\begin{array}{c}
q_{u p} \\
q_{l o} \\
T_{H t}
\end{array}\right] \text {. }} \\
& R_{f n t}=R_{a l}=\frac{L_{a l}}{k_{a l} \cdot A} \\
& R_{b c k}=R_{\text {tape }}+R_{\text {insl }}+R_{a l}+R_{\text {tape }} \\
& =2 \cdot R_{\text {tape }}+\frac{L_{i n s}}{k_{\text {ins }} \cdot A}+\frac{L_{a l}}{k_{a l} \cdot A},
\end{aligned}
$$

where $L_{m}$ and $L_{i n s}, k_{m}$ and $k_{i n s}$ are the thicknesses and thermal conductivies of the aluminum plate and Silica Aerogel insulation respectively. $A$ is the area of the panel perpendicular to the direction of heat flux.

\section{B Uncertainty Analysis}

Calculating a result $R_{0}$ based on measurements with independent variables $x_{i}$ where the result is described,

$$
R_{0}=f\left(x_{1}, x_{2}, \ldots, x_{L}\right),
$$

the uncertainties associated with the increase of each independent variable $R_{i}^{+}$are then

$$
R_{1}^{+}=f\left(x_{1}+u_{x 1}, x_{2}, \ldots, x_{L}\right)
$$




$$
\begin{aligned}
R_{2}^{+} & =f\left(x_{1}, x_{2}+u_{x 2}, \ldots, x_{L}\right) \\
R_{L}^{+} & =f\left(x_{1}, x_{2}, \ldots, x_{L}+u_{x L}\right) .
\end{aligned}
$$

The uncertainties for the decrease are calculated similarly, giving $R_{i}^{-}$. The contribution of each independent variable towards the uncertainty $\delta R_{i}$ is then

$$
\delta R_{i}=\frac{\delta R_{i}^{+}-\delta R_{i}^{-}}{2} \approx \Theta_{i} u_{i},
$$

where $\Theta_{i}$ is the general sensitivity index, reflecting how much changes in $x_{i}$ affect $R$. The resulting uncertainty is then

$$
u_{R}=\left[\sum_{i=1}^{L}\left(\delta R_{i}\right)^{2}\right]^{1 / 2}
$$

